\title{
Universality in Blow-Up for Nonlinear Heat Equations
}

\author{
J.Bricmont* \\ UCL, Physique Théorique, Louvain-la-Neuve, Belgium \\ A.Kupiainen ${ }^{\dagger}$ \\ Helsinki University, Mathematics Department, \\ Helsinki, Finland
}

\begin{abstract}
We consider the classical problem of the blowing-up of solutions of the nonlinear heat equation. We show that there exist infinitely many profiles around the blowup point, and for each integer $k$, we construct a set of codimension $2 k$ in the space of initial data giving rise to solutions that blow-up according to the given profile.
\end{abstract}

\section{Introduction}

We consider the problem of the blow-up of solutions of the initial value problem

$$
u_{t}=u_{x x}+u^{p}
$$

where $p>1, u=u(x, t), x \in \mathbf{R}$, and $u(\cdot, 0)=u_{0} \in C^{0}(\mathbf{R})$. It is well-known that, for a large class of initial data $u_{0}$, the solution will diverge in a finite time at a single point (for reviews on this problem, see [9, [13]).

We are interested in the profile of the solution at the time of blow-up. To explain what this means, let us fix the blow-up point to be 0 and the blow-up time to be $T$. Then, we ask whether it is possible to find a function $f^{*}(x)$ and a rescaling $g(t, T)$ so that

$$
\lim _{t \uparrow T}(T-t)^{\frac{1}{p-1}} u(g(t, T) x, t)=f^{*}(x)
$$

Moreover, we want to see how $g$ or $f^{*}$ depend on the initial data.

The prefactor $(T-t)^{\frac{1}{p-1}}$ in $(2)$ can be understood easily: for initial data $u_{0}(x)$ constant in $x, u(t)$ solves the ODE $\dot{u}=u^{p}$, i.e. $u(t)=((p-1)(T-t))^{\frac{1}{1-p}}$ for $T=$ $(p-1)^{-1} u_{0}^{1-p}$. We therefore expect that $f^{*}(0)=(p-1)^{\frac{1}{1-p}}$. However, we want to obtain $f^{*}$ for $x \neq 0$. In [10, 11, 12, 15] (see also [4, 5]) several possible $f^{*}$ 's are discussed,

${ }^{*}$ Supported by EC grant SC1-CT91-0695

${ }^{\dagger}$ Supported by NSF grant DMS-8903041 
and the set of initial data that will lead to a given $f^{*}$ is partially characterised. In the present paper, we shall show that there exists, in the space of initial data $C^{0}(\mathbf{R})$, sets $\mathcal{M}_{k}$ of codimension $2 k$, such that, for $u_{0} \in \mathcal{M}_{k}$, the limiting behaviour (2) is obtained, in the case $k=1$, for

$$
\begin{aligned}
g(t, T) & =((T-t)|\log (T-t)|)^{\frac{1}{2}} \\
f^{*}(x) & =\left(p-1+b^{*} x^{2}\right)^{\frac{1}{1-p}}
\end{aligned}
$$

where $b^{*}=\frac{(p-1)^{2}}{4 p}$, and in the case $k>1$ for

$$
\begin{aligned}
g(t, T) & =(T-t)^{\frac{1}{2 k}} \\
f_{b}^{*}(x) & =\left(p-1+b x^{2 k}\right)^{\frac{1}{1-p}} .
\end{aligned}
$$

where now $b$ is an arbitary positive number.

The lowest codimension 2 corresponds to fixing two parameters in the data that specify the blow-up point and time. To reach the other "strata", $2 k-2$ additional parameters describing the data need to be fixed.

Now we want to relate this problem to the renormalization group approach to the study of the asymptotic behaviour of solutions of nonlinear partial differential equations; this approach was initiated and developed in [1, 7, 8] and, from a mathematical point of view, in [3]. Although our actual proofs do not rely very much on this approach, the ideas used here are close to the ones of the renormalization group. In this approach, the long-time behaviour of the solution is related to the existence of fixed points of the renormalization group transformation (which basically amounts to solving the PDE over a finite time interval, and combining this with some scaling transformations). A given asymptotic behaviour is obtained, provided the initial data lie in the basin of attraction of a given fixed point. This basin of attraction is called a "universality class". In the simplest cases, the fixed points are stable but, in general, there can be one or more unstable or neutral directions for the renormalization group flow around the fixed point. This is exactly what happens here: $f^{*}$ and $f_{b}^{*}$ can be viewed as a fixed points of a renormalization group transformation having $2 k$ unstable ("relevant", in renormalization group terminology) directions. Thus, to converge towards the fixed point, one has to fine-tune $2 k$ parameters (one for each unstable direction) and this explains why $\mathcal{M}_{k}$ is of codimension $2 k$, and in what sense $f^{*}$ is "universal". In addition, we encounter also one neutral ("marginal") mode, which, for $k=1$, turns out to be stable when nonlinear effects are taken into account and for $k>1$ parametrizes a curve of fixed points. Thus, our result is also connected to the center manifold theory.

Our results are perturbative, i.e. the sets $\mathcal{M}_{k}$ consist of initial data that are close to the corresponding fixed point. Therefore, our results are similar to those of Bressan [2] who considers a nonlinearity $e^{u}$ instead of $u^{p}$ and obtains the universal profile analogous to our $k=1$ case. However, his method is different from ours and we obtain a control over the limit (2) which is uniform in $x$. 
To describe our main results, let us fix a positive number $T$, and introduce

$$
f(\xi)= \begin{cases}T^{\frac{1}{p-1}} u_{0}\left(T^{\frac{1}{2 k}} \xi\right) & k>1 \\ T^{\frac{1}{p-1}} u_{0}\left((|\log T| T)^{\frac{1}{2}} \xi\right) & k=1\end{cases}
$$

Let first $k=1$. We write the initial data as

$$
f(\xi)=f^{*}(\xi)\left(1+\frac{d_{0}+d_{1} \xi}{p-1+b^{*} \xi^{2}}\right)+g(\xi)
$$

where, given $g \in C^{0}(\mathbf{R}), d_{0}$ and $d_{1}$ are the two parameters to be fixed. We have

Theorem 1. There exists a $T_{0}>0$ such that, for each $0<T<T_{0}$ and $g \in C^{0}(\mathbf{R})$ with $\|g\|_{\infty}<\left(\log T_{0}\right)^{-2}$ one can find $d_{0}$ and $d_{1}$, such that the equation (1) with the initial data (7),(8) has a unique classical solution $u(x, t)$ on $\mathbf{R} \times[0, T)$ and

$$
\lim _{t \uparrow T}(T-t)^{\frac{1}{p-1}} u\left(((T-t)|\log (T-t)|)^{\frac{1}{2}} \xi, t\right)=f^{*}(\xi)
$$

uniformly in $\xi$ on $\mathbf{R}$.

Remark 1. We get a much more detailed information on $u(x, t)$, see the Proposition in Sect.3.

Remark 2. To leading order, $d_{0}(p-1)^{-\frac{p}{p-1}}=-\frac{a}{\log T}$, with $a=2 b^{*}(p-1)^{\frac{2 p-1}{1-p}}$, and is independent of $g$ (see Lemma 2 in Sect.3 below). This means that $d_{0}$ (and $d_{1}$ ) are nonzero, even if we have $g(\xi)=0$ in (8).

Remark 3. The proof can be extended to more general nonlinearities, than (1): we actually give below the proof for the equation

$$
u_{t}=u_{x x}+u^{p}+F(u)
$$

and we will assume that $F: \mathbf{R} \rightarrow \mathbf{R}$ is Lipschitz and satisfies

$$
|F(u)| \leq C\left(1+|u|^{q}\right)
$$

with $0 \leq q<p$, and

$$
\left|F\left(\lambda u_{1}\right)-F\left(\lambda u_{2}\right)\right| \leq C \lambda^{q}\left|u_{1}-u_{2}\right|
$$

for $\left|u_{1}\right|,\left|u_{2}\right| \leq 1$ and $\lambda \geq 1$. With little extra effort the proof extends to nonlinearities $F\left(u, u_{x}\right)$ in (1.10) that depend on $u_{x}$. We need then

$$
\left|F\left(u, u_{x}\right)\right| \leq C\left(1+|u|^{q}+\left|u_{x}\right|^{r}\right)
$$

with $q$ as in (11),

$$
r<\frac{2 p}{p+1},
$$

and the corresponding Lipschitz bound. 
Remark 4. The proof also extends to $x \in \mathbf{R}^{n}, n>1 ; d_{1} \xi$ in (8) becomes $\vec{d}_{1} \cdot \vec{\xi}$ where $\vec{d}_{1}$ is an $n$-component vector and $\xi^{2}$ becomes $\|\vec{\xi}\|^{2}$. Thus, we need to fix $n+1$ coefficients. For simplicity, we shall keep $n=1$.

For $k>1$, we take the data of the form

$$
f(\xi)=f_{b}^{*}(\xi)\left(1+\sum_{i=0}^{2 k-1} d_{i} \xi^{i}\left(p-1+b \xi^{2 k}\right)^{-1}\right)+g(\xi)
$$

where $d_{i}$ are now the parameters to be fixed, once a $g \in C^{0}(\mathbf{R})$ is given. We have then the

Theorem 2. There exist $T_{0}>0$ and $\varepsilon>0$ such that for $0<T<T_{0}$ and $g$ in $C^{0}(\mathbf{R})$ with $\|g\|_{\infty}<\varepsilon$ there are constants $d_{i} \in \mathbf{R}$ such that the equation (1) with the initial data (14) has a unique classical solution $u(x, t)$ on $\mathbf{R} \times[0, T)$ and

$$
\lim _{t \uparrow T}(T-t)^{\frac{1}{p-1}} u\left((T-t)^{\frac{1}{2 k}} \xi, t\right)=f_{b^{*}}^{*}(\xi)
$$

uniformly in $\xi$ on $\mathbf{R}$, for some $b^{*}>0$, where $b^{*} \rightarrow b$ as $\varepsilon \rightarrow 0$ and $T_{0} \rightarrow 0$.

Remark 1. Thus we have, for $k>1$, a line of fixed points $f_{b}^{*}$ and given initial data in the codimension $2 k$ set in $C^{0}(\mathbf{R})$, the $u(x, t)$ arrives to this line as $t \rightarrow T$. The only effect of the $g$ in the data (14) is to "renormalize" the $b$ occurring in the data. Compare with the $k=1$ case where there was a unique fixed point $f^{*}$.

Remark 2. Note that our assumptions, in both Theorems, allow initial data that are not everywhere positive.

Remark 3. Again, more general equations can be treated, but we leave that formulation for the reader.

\section{Dynamical systems formulation}

In this section we describe a change of variables that transforms the problem (1.10) into a problem of long time asymptotics. We also explain the main ideas of our proof.

We write (1.10) in the "blow-up-variables": given a $u: \mathbf{R} \times[0, T) \rightarrow \mathbf{R}$, define $\varphi: \mathbf{R} \times[-\log T, \infty) \rightarrow \mathbf{R}$ by

$$
u(x, t)=(T-t)^{-\frac{1}{p-1}} \varphi\left(\frac{x}{(T-t)^{1 / 2 k}},-\log (T-t)\right) .
$$

Then $u$ is a classical solution of (1.10) if and only if $\varphi(\xi, \tau)$ is a classical solution of

$$
\begin{aligned}
\dot{\varphi} & =L_{\tau}^{-2} \varphi^{\prime \prime}-\frac{1}{2 k} \xi \varphi^{\prime}-\frac{1}{p-1} \varphi+\varphi^{p}+F_{\tau}(\varphi) \\
\varphi\left(\xi, \tau_{0}\right) & =T^{\frac{1}{p-1}} u_{0}\left(T^{\frac{1}{2 k}} \xi\right)
\end{aligned}
$$


where $\tau_{0}=-\log T$,

$$
L_{\tau}=e^{\frac{1}{2} \tau(1-1 / k)} .
$$

and

$$
F_{\tau}(\varphi)=e^{-\frac{p}{p-1} \tau} F\left(e^{\frac{\tau}{p-1}} \varphi\right) .
$$

We will construct global solutions of (2), with suitable initial data thereby establishing blow-up for (1.10). Note that, for $k=1$, the scaling in (1) differs from the one used in (1.9) by a factor $\tau^{1 / 2}$.

Consider first the $k=1$ case with $F=0$. To understand the dynamics of (2), let us start by considering its linearization around the constant solution $\varphi=(p-1)^{\frac{1}{1-p}}$. The linear problem is $\dot{\phi}=\mathcal{L} \phi$, where

$$
\mathcal{L}=\partial^{2}-\frac{1}{2} \xi \partial+1 .
$$

with $\partial=\partial_{\xi}$. Hence, the first thing we have to do to understand the stability of the constant solution is to study the spectrum of the linear operator $\mathcal{L}$.

$\mathcal{L}$ is self-adjoint on $\mathcal{D}(\mathcal{L}) \subset L^{2}(\mathbf{R}, d \mu)$ with

$$
d \mu(\xi)=\frac{e^{-\xi^{2} / 4} d \xi}{\sqrt{4 \pi}}
$$

The spectrum of $\mathcal{L}$ is

$$
\operatorname{spec}(\mathcal{L})=\left\{1-\frac{n}{2} \mid n \in \mathbf{N}\right\}
$$

and we take as eigenfunctions multiples of Hermite polynomials

$$
h_{n}(\xi)=\sum_{m=0}^{\left[\frac{n}{2}\right]} \frac{n !}{m !(n-2 m) !}(-1)^{m} \xi^{n-2 m}
$$

that satisfy

$$
\int h_{n} h_{m} d \mu=2^{n} n ! \delta_{n m}
$$

and

$$
\mathcal{L} h_{n}=\left(1-\frac{n}{2}\right) h_{n} .
$$

Thus the derivative of the RHS of (2) at the constant solution has 2 expanding ("relevant") directions and one neutral ("marginal") one, $h_{2}=\xi^{2}-2$.

How do we understand now the emergence of the fixed point $f^{*}$ ? We get a clue on what should happen by considering the following scaling: let

$$
\varphi_{L}(\xi, \tau)=\varphi\left(L \xi, L^{2} \tau\right)
$$

$\varphi_{L}$ satisfies the equation

$$
H_{1}\left(\varphi_{L}\right)=L^{-2}\left(-\dot{\varphi}_{L}+\varphi_{L}^{\prime \prime}\right)+F_{L^{2} \tau}\left(\varphi_{L}\right) .
$$


where we defined

$$
H_{k}(\varphi)=\frac{1}{2 k} \xi \partial_{\xi} \varphi+\frac{1}{p-1} \varphi-\varphi^{p} .
$$

Hence, as $L \rightarrow \infty$, we expect the solutions of $H_{k}(\varphi)=0$ (for $k=1$ ) to be relevant. These are, for any $k$, given by the one-parameter family $f_{b}^{*}$ (see (1.6)). Before we explain why only one $b^{*}$ is selected, we will compare the above with the $k>1$ case.

For $k>1$, as $\tau \rightarrow \infty$, we expect the solution of

$$
\dot{\varphi}=-\frac{1}{2 k} \xi \varphi^{\prime}-\frac{1}{p-1} \varphi+\varphi^{p}
$$

to be relevant $($ see $(2,4))$. This can of course be integrated in closed form, but before doing that, let us first look at its linearization around the constant solution $\varphi=(p-$ $1)^{\frac{1}{1-p}}$. The linear problem is $\dot{\phi}=\mathcal{L}_{\infty} \phi$, where

$$
\mathcal{L}_{\infty}=-\frac{1}{2 k} \xi \partial+1
$$

and so, e.g. in the space of polynomials, we have now $2 k$ expanding directions corresponding to $\xi^{n}$, for $n<2 k$.

Equation (15) is solved by putting $\varphi(\xi, \tau)=e^{-\frac{\tau}{p-1}} h\left(e^{-\tau / 2 k} \xi, \tau\right)$ whereby $\partial_{\tau} h(y, \tau)=$ $e^{-\tau} h(y, \tau)^{p}$ and so, for $\rho=\tau-\tau_{0}$

$$
\varphi(\xi, \tau)=\frac{e^{-\frac{\rho}{p-1}} f\left(e^{-\rho / 2 k} \xi\right)}{\left[1-(p-1) f\left(e^{-\rho / 2 k} \xi\right)^{p-1}\left(1-e^{-\rho}\right)\right]^{1 / p-1}}
$$

where $\varphi\left(\xi, \tau_{0}\right)=f(\xi)$. Depending on $f,(17)$ has several possible asymptotics as $\rho \rightarrow \infty$. In the space of constant $f$ 's we have the stable $f=0$ and unstable $f=(p-1)^{-\frac{1}{p-1}}$ fixed points. The latter is stable in a suitable codimension $2 k$ space: let us consider say $f$ smooth,

$$
f(0)=(p-1)^{-\frac{1}{p-1}}, f^{(\ell)}(0)=0 \quad \ell<2 k, f^{(2 k)}(0)=\beta<0
$$

and

$$
0 \leq f(\xi)<(p-1)^{-\frac{1}{p-1}} \quad \xi \neq 0
$$

Then, for all $\xi \in \mathbf{R}$

$$
\left|\varphi(\xi, \tau)-f_{b}(\xi)\right| \underset{\tau \rightarrow \infty}{\longrightarrow} 0
$$

where

$$
f_{b}(\xi)=\left(p-1+b \xi^{2 k}\right)^{-\frac{1}{p-1}}
$$

for some $b$ depending on $\beta, k, p$.

These considerations thus lead us to expect (2) to have global solutions with initial data in a suitable codimension $2 k$ set in a ball around (21) in a suitable Banach space. Note however, that the perturbation $L_{\tau}^{-2} \varphi_{\xi \xi}$ in (2) is a very singular one: we certainly need to keep track of its smoothing effects. On the other hand, we want to retain as much as possible of the nice picture obtained above in the $\tau \rightarrow \infty$ limit. We explain now 
how this is done for (2) linearized around the constant solution, leaving the nonlinear analysis to the actual proof in Section 4.

The linearization of (2) around the constant solution is $\dot{\phi}=\mathcal{L}_{\tau} \phi$ where

$$
\mathcal{L}_{\tau}=L_{\tau}^{-2} \partial_{\xi}^{2}-\frac{1}{2 k} \xi \partial_{\xi}+1
$$

In order to study linear stability, we thus need some properties of the fundamental solution $K_{\tau \sigma}$ of $(22)$, i.e.

$$
\partial_{\tau} K_{\tau \sigma}=\mathcal{L}_{\tau} K_{\tau \sigma}, \quad K_{\sigma \sigma}=\mathrm{id}
$$

$K_{\tau \sigma}$ is conveniently found, by conjugating the problem (23) to a time independent one:

$$
K_{\tau \sigma}=S_{\tau} e^{(\tau-\sigma) \mathcal{L}} S_{\sigma}^{-1}
$$

where

$$
\left(S_{\tau} \theta\right)(\xi)=\theta\left(L_{\tau} \xi\right)
$$

and $\mathcal{L}$ is given in (6). Thus, in terms of kernels

$$
K_{\tau \sigma}\left(\xi, \xi^{\prime}\right)=L_{\sigma} e^{(\tau-\sigma) \mathcal{L}}\left(L_{\tau} \xi, L_{\sigma} \xi^{\prime}\right)
$$

and, since the kernel of $e^{\rho \mathcal{L}}$ is given explicitely by Mehler's formula 14:

$$
e^{\rho \mathcal{L}}\left(\xi, \xi^{\prime}\right)=\left[4 \pi\left(1-e^{-\rho}\right)\right]^{-1 / 2} e^{\rho} \exp \left[-\frac{\left(\xi e^{-\rho / 2}-\xi^{\prime}\right)^{2}}{4\left(1-e^{-\rho}\right)}\right]
$$

(26) can be written in the form

$$
K_{\tau \sigma}\left(\xi, \xi^{\prime}\right)=e^{\rho} \delta_{L^{2}}\left(e^{-\rho / 2 k} \xi-\xi^{\prime}\right)
$$

where $\rho=\tau-\sigma, L^{2}=L_{\sigma}^{2}\left(1-e^{-\rho}\right)^{-1}$, and

$$
\delta_{L^{2}}(\xi)=\frac{L}{\sqrt{4 \pi}} e^{-L^{2} \xi^{2} / 4}
$$

(27) and (28) show clearly that the effect of the $L_{\tau}^{-2} \partial_{\xi}^{2}$ is to smoothen the kernel of the linear problem (see (16)), $\dot{\varphi}=\left(1-\frac{1}{2 k} \xi \partial_{\xi}\right) \varphi$, which is just

$$
K_{\rho}^{\infty}\left(\xi, \xi^{\prime}\right) \equiv e^{\rho} \delta\left(e^{-\rho / 2 k} \xi-\xi^{\prime}\right)
$$

i.e. the distributional limit of (27) as $L \rightarrow \infty$ i.e. as $\sigma \rightarrow \infty$.

As in the $k=1$ case, we may now study the stability of the linearization in a Hilbert space. The "eigenfunctions" of $K_{\tau \sigma}$ are readily obtained From (9) by the conjugation (24). We put

$$
h_{n}(\xi, \tau)=L_{\tau}^{-n} h_{n}\left(L_{\tau} \xi\right)=\sum_{m=0}^{\left[\frac{n}{2}\right]} \frac{n !}{m !(n-2 m) !}\left(-L_{\tau}^{-2}\right)^{m} \xi^{n-2 m}
$$


(note that $h_{n} \rightarrow \xi^{n}$ as $\tau \rightarrow \infty$ ) whereby $h_{n}(\cdot, \tau)$ form a basis of $L^{2}\left(\mathbf{R}, d \mu_{\tau}\right)$ where

$$
d \mu_{\tau}(\xi)=\frac{L_{\tau}}{\sqrt{4 \pi}} e^{-L_{\tau}^{2} \xi^{2} / 4} d \xi
$$

and

$$
\left(h_{n}(\cdot, \tau), h_{m}(\cdot, \tau)\right)_{\tau}=\int h_{n}(\xi, \tau) h_{m}(\xi, \tau) d \mu_{\tau}(\xi)=L_{\tau}^{-2 n} 2^{n} n ! \delta_{n m}
$$

We then have

$$
K_{\tau \sigma} h_{n}(\cdot, \sigma)=e^{(\tau-\sigma)(1-n / 2 k)} h_{n}(\cdot, \tau)
$$

which should be compared with $K_{\rho}^{\infty} p_{n}=e^{\rho\left(1-\frac{n}{2 k}\right)} p_{n}$ for $p_{n}(\xi)=\xi^{n}$. The $h_{n}$ with $n<2 k$ form thus a convenient basis for the expanding modes.

Finally we want to comment on the effect of the nonlinear terms. The linear analysis presented above deals with deviations from the constant solution and turns out to describe the solution well for $|\xi|$ not too large. We thus need to understand why the fixed points $f_{b}^{*}$ are selected, and, for $k=1$, why only one $b^{*}$ occurs. Finally we need to understand the stability problem for $|\xi|$ large. We shall only discuss here the $k=1$ case, since $k>1$ is actually easier (see Section 4 ).

Consider $k=1$. We introduce

$$
\varphi_{b}(\xi, \tau)=\left(p-1+b \xi^{2} / \tau\right)^{\frac{1}{1-p}}
$$

where the factor $\tau$ can be understood by comparing the scaling in (1) and in (1.9), and we study the flow near $\varphi_{b}$. Let us rewrite (2) in terms of $\eta$, where

$$
\varphi(\xi, \tau)=\varphi_{b}(\xi, \tau)+\eta(\xi, \tau)
$$

We get, using $H_{1}\left(\varphi_{b}\right)=0$ (see $\left.(14)\right)$,

$$
\begin{aligned}
\dot{\eta} & =\eta^{\prime \prime}-H_{1}\left(\varphi_{b}+\eta\right)+H_{1}\left(\varphi_{b}\right)+\varphi_{b}^{\prime \prime}-\dot{\varphi}_{b}+F_{\tau}\left(\varphi_{b}+\eta\right) \\
& =(\mathcal{L}+W) \eta+M(\eta)+\varphi_{b}^{\prime \prime}-\dot{\varphi}_{b}+F_{\tau}\left(\varphi_{b}+\eta\right)
\end{aligned}
$$

where we write $\frac{-1}{p-1}=1-\frac{p}{p-1}$, and introduce

$$
\begin{aligned}
& W=p\left(\varphi_{b}^{p-1}-\frac{1}{p-1}\right) \\
& M(\eta)=\left(\varphi_{b}+\eta\right)^{p}-\varphi_{b}^{p}-p \varphi_{b}^{p-1} \eta .
\end{aligned}
$$

$\mathcal{L}$, given by (6), has two unstable modes. Note that, formally, (i.e., for $\xi$ of order one) $W$ is $\mathcal{O}\left(\tau^{-1}\right), M$ is nonlinear in $\eta$ and $\varphi_{b}^{\prime \prime}-\varphi_{b}^{\prime}$ is $\mathcal{O}\left(\tau^{-1}\right)$. Our goal will be to construct a center manifold for (36), i.e. to find the parameters $d_{0}, d_{1}$ in (1.8), such that the flow of (36) stays bounded.

To explain the idea of the proof we first consider the special case $p=2$ and $\eta$ even in $\xi$, which will imply $d_{1}=0$ in (1.8). This example contains all the relevant features of the general case. Now,

$$
\varphi_{b}(\xi, \tau)=\left(1+b \xi^{2} / \tau\right)^{-1}
$$


It is convenient to first find $d_{0}$ approximately, exact to order $1 / \tau$. Let

$$
\eta_{0}(\tau)=\frac{a}{\tau}
$$

and define $\psi$ by

$$
\eta=\eta_{0}+\psi
$$

Then $\psi$ satisfies the equation

$$
\dot{\psi}=(\mathcal{L}+V) \psi+N(\psi)+\alpha
$$

with (for later purpose we write this for general $p$ )

$$
\begin{aligned}
V & =p\left(\left(\varphi_{b}+\eta_{0}\right)^{p-1}-\frac{1}{p-1}\right) \\
N(\psi) & =\left(\varphi_{b}+\eta_{0}+\psi\right)^{p}-\left(\varphi_{b}+\eta_{0}\right)^{p}-p\left(\varphi_{b}+\eta_{0}\right)^{p-1} \psi+F_{\tau}\left(\varphi_{b}+\eta_{0}+\psi\right) \\
\alpha & =\varphi_{b}^{\prime \prime}-\dot{\varphi}_{b}+(\mathcal{L}+W) \eta_{0}-\dot{\eta}_{0}+M\left(\eta_{0}\right) \\
& =\varphi_{b}^{\prime \prime}-\dot{\varphi}_{b}+\eta_{0}+W \eta_{0}-\dot{\eta}_{0}+M\left(\eta_{0}\right) .
\end{aligned}
$$

We shall see how to choose $a$ and $b$ so that the flow of $\psi$ in (41) can remain bounded. Let us decompose $\psi$ as

$$
\psi=\psi_{0}(\tau)+\psi_{2}(\tau) h_{2}+\psi^{\perp}
$$

where $\psi^{\perp}$ is orthogonal to $h_{n}, n \leq 2$ (we will later in the actual proof refine (45)). Next we expand $V$ and $\alpha$ (for $\xi=\mathcal{O}(1)$ ):

$$
\begin{aligned}
& V=-\frac{2 b \xi^{2}}{\tau}+\frac{2 a}{\tau}+\mathcal{O}\left(\frac{1}{\tau^{2}}\right) \\
& \left.\alpha=(a-2 b) \tau^{-1}+\left(a+a^{2}+\left(12 b^{2}-b-2 a b\right) \xi^{2}\right)\right) \tau^{-2}+\mathcal{O}\left(\tau^{-3}\right) .
\end{aligned}
$$

Inserting (40), (45) in (41) and retaining only the leading terms in $1 / \tau$ and $\psi_{i}, i=0,2$, we get from $\dot{\psi}_{i}=\left(2^{i} i !\right)^{-1}\left(h_{i}, \dot{\psi}\right)\left((\cdot, \cdot)\right.$ is the scalar product of $\left.L^{2}(\mathbf{R}, d \mu)\right)$ :

$$
\begin{aligned}
& \dot{\psi}_{0}=\psi_{0}+(a-2 b) \tau^{-1}+R_{0} \\
& \dot{\psi}_{2}=\beta \tau^{-1} \psi_{2}+\left(12 b^{2}-b-2 a b\right) \tau^{-2}+R_{2}
\end{aligned}
$$

where $R_{0}=\mathcal{O}\left(\tau^{-2}+\tau^{-1}|\psi|+|\psi|^{2}\right), R_{2}=\mathcal{O}\left(\tau^{-3}+\tau^{-1}\left|\psi_{0}\right|+\tau^{-2}\left|\psi_{2}\right|+|\psi|^{2}\right)$, and $\beta=$ $2 a-\frac{1}{4} b\left(\xi^{2} h_{2}, h_{2}\right)=2 a-20 b$ (coming from the $V \psi$ term in (41)). We choose now $a$ so that the $\mathcal{O}\left(\tau^{-1}\right)$ term in $\dot{\psi}_{0}$ vanishes i.e.

$$
a=2 b
$$

and $b$ such that the $\mathcal{O}\left(\tau^{-2}\right)$ term in $\dot{\psi}_{2}$ is zero:

$$
b=b^{*}=1 / 8 .
$$


Note that this choice correspond to $b=b^{*}$ in (1.4) for $p=2$ and $a$ as in Remark 1.2. Then $\beta=-2$ and our equations read

$$
\dot{\psi}_{0}=\psi_{0}+R_{0}, \quad \dot{\psi}_{2}=-\frac{2}{\tau} \psi_{2}+R_{2}
$$

Now,

$$
\psi_{0}=\mathcal{O}\left(\tau^{-2}\right), \quad \psi_{2}=\mathcal{O}\left((\log \tau) \tau^{-2}\right)
$$

would be consistent solutions. Of course, we need to show that the expanding variable $\psi_{0}$ will satisfy $(53)$ by a suitable choice of $\psi_{0}\left(\tau_{0}\right)$, i.e. of the parameter $d_{0}$ in Theorem 1 . This is rather easy to do, using the fact that $\psi_{0}$ is expanding; in the general case (with $\left.d_{1} \neq 0\right)$, we shall use a topological argument.

If we were to expand $\psi^{\perp}$ in $(45)$ as

$$
\psi^{\perp}=\sum_{i=2}^{\infty} \psi_{2 i} h_{2 i}
$$

we would then formally get

$$
\dot{\psi}_{2 i}=-(i-1) \psi_{2 i}+\mathcal{O}\left(1 / \tau^{1+i}\right)+N(\psi)_{2 i}
$$

(in $\alpha$, we have an extra factor of $\tau^{-1}$ coming from the derivatives or from $\eta_{0}$ ) and the formal solution would be

$$
\psi_{2 i}(\tau)=\mathcal{O}\left(\tau^{-1-i}\right)
$$

so that $\psi_{2 i}(\tau) h_{2 i}\left(\xi \tau^{1 / 2}\right) \rightarrow 0$ as $\tau \rightarrow \infty$, for all $i$ (to prove (1.9), we need to scale $\xi$ here by $\tau^{1 / 2}$, see $\left.(1)\right)$. However, (54) will not be a good representation for large $\xi$ and we need to proceed differently.

We decompose $\psi$ to a part localized on an interval around the origin and to a part describing the large $|\xi|$ behaviour. For this let $\chi \in C_{0}^{\infty}(\mathbf{R})$ be non-negative, $\chi=1$ on $[-1,1]$ and $\chi=0$ on $[-2,2]^{c}$. Let $K>0$, and put

$$
\chi(\xi, \tau)=\chi\left(\left(K \tau^{1 / 2}\right)^{-1} \xi\right) .
$$

$K$ will be taken suitably large, see below. Let now

$$
\psi=\psi \chi+\psi(1-\chi) \equiv \psi_{s}+\psi_{l}
$$

The "small $\xi$ part", $\psi_{s}$, will be decomposed as above:

$$
\psi_{s}(\xi, \tau)=\psi_{0}(\tau)+\psi_{2}(\tau) h_{2}(\xi)+\psi^{\perp}(\xi, \tau)
$$

and we shall prove that

$$
\begin{aligned}
\left|\psi_{0}(\tau)\right| & \leq C \tau^{-2} \\
\left|\psi_{2}(\tau)\right| & \leq \tau^{-2+\delta} \\
\left|\psi^{\perp}(\xi, \tau)\right| & \leq C\left(1+|\xi|^{3}\right) \tau^{-2} \\
\left\|\psi_{l}(\cdot, \tau)\right\|_{\infty} & \leq C \tau^{-1 / 2} .
\end{aligned}
$$


for any $\delta>0$. This bound on $\psi_{2}$ is a convenient upper bound on the $(\log \tau) \tau^{-2}$ behaviour which is expected on the basis of $(52,53)$. Note that $\psi_{0}, \psi_{2}$ are functions only of $\tau$, while $\psi^{\perp}, \psi_{l}$ depend on $\tau$ and $\xi$.

The detailed $\psi_{l}$ bound will be explained in the proof below, but here we want to comment only on the decay in $\xi$ that we expect. The reason that (54) is not a good expansion is that the eigenfunctions of $\mathcal{L}$, i.e. $h_{i}$, grow at infinity; the more they are contracted by $e^{\tau \mathcal{L}}$, the more they grow. This would make the nonlinear term in (41) impossible to control. However, for $|\xi|>K \tau^{1 / 2}$, the $V$ in (42) (see (39)) is not any more small; actually $\mathcal{L}+V$ behaves like $\mathcal{L}-\frac{p}{p-1}$ in that region and this operator has purely negative spectrum. This is why an $L^{\infty}$-bound such as (63) will hold.

The proof of the general $p$ case is very similar. We have now 2 expanding modes (if $\eta$ is not even), and the number $b$ is again determined from a condition that the neutral mode contracts like $\tau^{-2}$ (with possibly logarithmic corrections).

\section{The proof, $k=1$}

Theorem 1 reads, in terms of $\varphi$, as

Theorem 3. There exists a $T_{0}>0$ such that, for each $0<T<T_{0}$ and $g \in C^{0}(\mathbf{R})$ with $\|g\|_{\infty}<\left(\log T_{0}\right)^{-2}$ one can find $d_{0}$ and $d_{1}$, such that the equation (2.2) with the initial data $(2.3,1.7,1.8)$ has a unique classical solution $\varphi(\xi, \tau)$ satisfying

$$
\lim _{\tau \rightarrow \infty}\left\|\varphi\left(\cdot \tau^{1 / 2}, \tau\right)-f^{*}(\cdot)\right\|_{\infty}=0
$$

We consider the equation for $\psi$ given by (2.41)-(2.44). The initial data is given by (see $(2.3),(1.7),(1.8),(2.35),(2.40))$

$$
\psi\left(\xi, \tau_{0}\right)=\varphi_{b^{*}}\left(\xi, \tau_{0}\right)\left(\frac{d_{0}+d_{1} \xi \tau_{0}^{-1 / 2}}{p-1+b^{*} \xi^{2} / \tau_{0}}\right)-a \tau_{0}^{-1}+g\left(\xi \tau_{0}^{-1 / 2}\right)
$$

Next we state the properties of $\psi$ that we want to establish. We write $\psi$ as in (2.58),

$$
\psi=\psi_{s}+\psi_{l}
$$

with this time

$$
\psi_{s}(\xi, \tau)=\sum_{m=0}^{2} \psi_{m}(\tau) h_{m}(\xi)+\psi^{\perp}(\xi, \tau)
$$

We will prove the

Proposition. With the assumptions of the Theorem, for any $\delta>0$, there exist a $\tau_{0}$ and constants $d_{0}, d_{1}$, such that $\psi$, given by (2),(3) will satisfy

$$
\left|\psi_{m}(\tau)\right| \leq \begin{cases}A \tau^{-2} & m=0,1 \\ \tau^{-2+\delta} & m=2\end{cases}
$$




$$
\left|\psi^{\perp}(\xi, \tau)\right| \leq A\left(1+|\xi|^{3}\right) \tau^{-2}
$$

and

$$
\left\|\psi_{l}\right\|_{\infty} \leq A_{l} \tau^{-\frac{1}{2}}
$$

for some constants $A, A_{l}$, uniformly on $\left[\tau_{0}, \infty\right)$.

Remark. Theorem 3 follows immediately from the Proposition, which implies that $\left\|\psi\left(\cdot \tau^{1 / 2}, \tau\right)\right\|_{\infty} \leq \mathcal{O}\left(\tau^{-1 / 2}\right.$ ) (by $(2.57),|\xi|$ in $\psi_{s}$ is bounded by $2 K \tau^{1 / 2}$ ). The $\delta$ in (4) may be made arbitary small by increasing $\tau_{0}$ (i.e. decreasing the data in $\psi$ or, equivalently, taking $T$ small and $u_{0}$ large in (2.3)). It will be convenient in the proof to distinguish between $A$ and $A_{l}$.

Proof. Let us assume that (4)-(6) hold for some $\sigma \geq \tau_{0}$ and study the existence and properties of the solution for subsequent times on an interval $[\sigma, \sigma+\rho]$. We shall choose below a sufficiently large constant $\rho$, and prove iteratively our bounds on intervals of the form $\left[\tau_{n}, \tau_{n+1}\right]$ with $\tau_{n}=\tau_{0}+n \rho$.

To prove existence and uniqueness, write (2.41) as an integral equation

$$
\psi(\tau)=K(\tau, \sigma) \psi(\sigma)+\int_{\sigma}^{\tau} d s K(\tau, s)[N(\psi(s))+\alpha(\cdot, s)]
$$

for $\psi(\tau) \equiv \psi(\cdot, \tau) . K$ is the fundamental solution of the linear equation $\dot{K}=(\mathcal{L}+V) K$. We study the three terms in (7) separately.

We expand the linear term in $\psi$ as in (2) and (3):

$$
K(\tau, \sigma) \psi(\sigma)=\sum_{m=0}^{2} \theta_{m} h_{m}+\theta^{\perp}+\theta_{l}
$$

with

$$
\theta_{l}(\xi, \tau)=(1-\chi(\xi, \tau))(K(\tau, \sigma) \psi(\sigma))(\xi) .
$$

Lemma 1 collects the bounds for the $\theta$ 's:

Lemma 1. For any $\rho>0$, there exists a $\tau_{0}$ such that, if $\psi(\sigma)$ satisfies (4)-(6) for $\sigma \geq \tau_{0}$, then, for $\tau \leq \sigma+\rho$,

$$
\begin{aligned}
\left|\theta_{m}(\tau)-e^{\left(1-\frac{m}{2}\right)(\tau-\sigma)} \psi_{m}(\sigma)\right| & \leq(\tau-\sigma) C \tau^{-3+\delta} \quad m=0,1 \\
\left|\theta_{2}(\tau)-\left(\frac{\sigma}{\tau}\right)^{2} \psi_{2}(\sigma)\right| & \leq(\tau-\sigma) C A \tau^{-3} \\
\left|\theta^{\perp}(\xi, \tau)\right| & \leq C\left(e^{-\frac{1}{2}(\tau-\sigma)} A+e^{-(\tau-\sigma)^{2}} A_{l}\right)\left(1+|\xi|^{3}\right) \tau^{-2} \\
\left\|\theta_{l}(\cdot, \tau)\right\|_{\infty} & \leq C\left(A_{l} e^{-\frac{(\tau-\sigma)}{p}}+A e^{(\tau-\sigma)}\right) \tau^{-\frac{1}{2}}
\end{aligned}
$$

Here and below we use $C$ or $c$ to denote a generic constant, which may vary from place to place. $C$ may depend on $K$ in (2.57), but not on $A, A_{l}$ or anything else (unless explicitely stated otherwise), and, since we shall consider $K$ as fixed, but sufficiently large, these constants are fixed also. 
For the $\alpha$-term, we need to specify $\eta_{0}$, i.e. the number $a$ in $(2.40)$, as well as $b^{*}$, so that the contribution of $\alpha$ to $\psi$ is (almost) of the same order of magnitude as the bounds (10)-(13). We have

Lemma 2. Let

$$
a=2 b^{*}(p-1)^{\frac{2 p-1}{1-p}}=\frac{(p-1)^{\frac{1}{1-p}}}{2 p}
$$

i.e. $b^{*}=\frac{(p-1)^{2}}{4 p}$ and set $b=b^{*}$ in (2.44). Define

$$
\mathcal{A}(\xi, \tau, \sigma)=\int_{\sigma}^{\tau} d s K(\tau, s) \alpha(\cdot, s)
$$

Then $\mathcal{A}(\xi, \tau, \sigma)$ has an expansion as in $(8)$ :

$$
\mathcal{A}=\sum_{m=0}^{2} \mathcal{A}_{m} h_{m}+\mathcal{A}^{\perp}+\mathcal{A}_{l}
$$

with

$$
\begin{aligned}
\left|\mathcal{A}_{m}(\tau, \sigma)\right| & \leq(\tau-\sigma) C e^{(\tau-\sigma)} \tau^{-2} \quad m=0,1 \\
\left|\mathcal{A}_{2}(\tau, \sigma)\right| & \leq(\tau-\sigma) C \tau^{-3} \\
\left|\mathcal{A}^{\perp}(\xi, \tau, \sigma)\right| & \leq(\tau-\sigma) C\left(1+|\xi|^{3}\right) \tau^{-2} \\
\left\|\mathcal{A}_{l}(\cdot, \tau, \sigma)\right\|_{\infty} & \leq(\tau-\sigma) C e^{(\tau-\sigma)} \tau^{-\frac{1}{2}}
\end{aligned}
$$

Given Lemmas 1 and 2, we may next solve (7) by the contraction mapping principle. Thus, write (7) as

$$
\psi(\tau)=\psi^{0}(\tau)+\mathcal{N}(\psi, \tau) \equiv \mathcal{S}(\psi, \tau)
$$

where $\psi^{0}$ collects the linear and inhomogenous terms that were bounded in Lemmas 1 and 2 .

Consider now the following norm on $C^{0}(\mathbf{R})$. For $\psi \in C^{0}(\mathbf{R})$, we set

$$
|\psi|_{\tau}=\tau^{2-\delta}\left\|\left(1+|\xi|^{3}\right)^{-1} \chi \psi\right\|_{\infty}+\tau^{\frac{1}{2}-\delta}\|(1-\chi) \psi\|_{\infty}
$$

where $\chi=\chi(\cdot, \tau)$. We have

$$
C_{1}(\tau)\|\psi\|_{\infty} \leq|\psi|_{\tau} \leq C_{2}(\tau)\|\psi\|_{\infty}
$$

for $C_{1}(\tau)>0$ and thus $C^{0}(\mathbf{R})$ is complete in the norm $\left.|\cdot|_{\tau}\right)$.

Equation (20) is now solved for $\psi(\tau) \in C^{0}(\mathbf{R})$ for $\tau \in[\sigma, \sigma+\rho]$, with the norm

$$
\|\psi\|_{\rho}=\sup _{\tau \in[\sigma, \sigma+\rho]}|\psi(\tau)|_{\tau}
$$

We shall choose below $\rho$ large enough and then take $\tau_{0}$ so that, for $\sigma \geq \tau_{0}$, we have, $\frac{\tau}{\sigma} \leq 1+\frac{\rho}{\sigma} \leq 2, e^{c \rho} \leq \tau_{0}^{\delta}$ and $A, A_{l} \leq \tau_{0}^{\delta}$. Then, it is an immediate corollary of Lemmas 1 and 2 , that

$$
\left\|\psi^{0}\right\|_{\rho} \leq C
$$


and we shall prove

Lemma 3. $\mathcal{S}$ maps the ball

$$
B_{0}=\left\{\psi \in C^{0}(\mathbf{R}) \mid\left\|\psi-\psi^{0}\right\|_{\rho} \leq \rho \tau^{-2 \delta}\right\}
$$

into itself and, for $\psi_{1}, \psi_{2} \in B_{0}$,

$$
\left\|\mathcal{S}\left(\psi_{1}\right)-\mathcal{S}\left(\psi_{2}\right)\right\|_{\rho} \leq \lambda\left\|\psi_{1}-\psi_{2}\right\|_{\rho}
$$

with $\lambda<1$. Moreover, for $\psi \in B_{0}$, we can write

$$
\mathcal{N}(\psi, \tau)=\sum_{m=0}^{2} \beta_{m} h_{m}+\beta^{\perp}+\beta_{l}
$$

where, for $\tau \in[\sigma, \sigma+\rho]$,

$$
\begin{aligned}
\left|\beta_{m}(\tau)\right| & \leq(\tau-\sigma) \tau^{-2} \quad m=0,1 \\
\left|\beta_{2}(\tau)\right| & \leq(\tau-\sigma) \tau^{-3} \\
\left|\beta^{\perp}(\xi, \tau)\right| & \leq(\tau-\sigma)\left(1+|\xi|^{3}\right) \tau^{-2} \\
\left\|\beta_{l}(\cdot, \tau)\right\|_{\infty} & \leq(\tau-\sigma) \tau^{-\frac{1}{2}}
\end{aligned}
$$

Remark. Using the Lemmas, it is straightforward to show that (7) has a $C^{0}$ solution $\psi$. Using integration by parts and the regularity of the kernel $K(\tau, \sigma)$ (see $(41,44)$ below), one can show that this solution is actually smooth and is the unique classical solution of equation (2.41).

With Lemmas 1-3 we may now prove the Proposition. First, writing $\psi=\psi^{0}+\psi^{1}$, we have the bounds (28-31) for $\psi^{1}$ and thus, combining these with (10)-(13), (16)-(19), we get the following estimates for the flow, for $\tau \leq \sigma+\rho, \sigma \geq \tau_{0}$ :

$$
\begin{aligned}
& \left|\psi_{m}(\tau)-e^{\left(1-\frac{m}{2}\right)(\tau-\sigma)} \psi_{m}(\sigma)\right| \leq(\tau-\sigma) C e^{(\tau-\sigma)} \tau^{-2} \quad m=0,1 \\
& \left|\psi_{2}(\tau)-\left(\frac{\sigma}{\tau}\right)^{2} \psi_{2}(\sigma)\right| \leq(\tau-\sigma) C A \tau^{-3} \\
& \left|\psi^{\perp}(\xi, \tau)\right| \leq C\left(e^{-\frac{1}{2}(\tau-\sigma)} A+e^{-(\tau-\sigma)^{2}} A_{l}+(\tau-\sigma)\right)\left(1+|\xi|^{3}\right) \tau^{-2} \\
& \left\|\psi_{l}(\cdot, \tau)\right\|_{\infty} \leq C\left(A_{l} e^{-\frac{(\tau-\sigma)}{p}}+A e^{(\tau-\sigma)}+(\tau-\sigma) e^{(\tau-\sigma)}\right) \tau^{-\frac{1}{2}}
\end{aligned}
$$

Now, we use (32-35) to prove the Proposition inductively. First, we prove the bounds (4-6) for all times of the form $\tau_{n}=\tau_{0}+n \rho, n \geq 0$, with some constants $\tilde{A}, \tilde{A}_{l}$. Then, it is easy to get (4-6) from (32-35) with $\sigma=\tau_{n}$, with possibly other constants, depending only on $\rho$, for all times (for $m=2$, one uses inequality (38) below).

Next, we observe that, for $n=0, \tau=\tau_{0}, \psi$ is given by (1) and $\|g\|_{\infty} \leq \tau_{0}^{-2}$. We have

$$
\left|\psi_{0}\left(\tau_{0}\right)+\frac{a}{\tau_{0}}-d_{0} \gamma_{0}\right|+\left|\psi_{1}\left(\tau_{0}\right)-d_{1} \gamma_{1} \tau_{0}^{-1 / 2}\right| \leq C \tau_{0}^{-2}
$$


for nonzero constants $\gamma_{0}, \gamma_{1}$. From (1) and (36), it is easy to see that (4)-(6) hold for $n=0, \tau=\tau_{0}$ for a suitable choice of $d_{0}, d_{1}$. Actually, one also sees from (36) that we may, instead of varying $d_{m}$ vary $\psi_{m}\left(\tau_{0}\right)$. Let $\psi_{m}\left(\tau_{0}\right)$ be in the interval $\left[-\tilde{A} \tau_{0}^{-2}, \tilde{A} \tau_{0}^{-2}\right]$ for $\tilde{A}$ large. Let us assume now that we can find $\psi_{m}\left(\tau_{0}\right)$ in that interval, such that (4) holds for $m=0,1$, for all times, with $A$ replaced by $\tilde{A}$. Then, $(5,6)$ hold, using $(34,35)$ : choose $\rho$ large enough so that

$$
\begin{gathered}
C\left(\tilde{A} e^{-\rho / 2}+\tilde{A}_{l} e^{-\rho^{2}}+\rho\right) \leq \tilde{A} \\
C\left(\tilde{A}_{l} e^{-\rho / p}+\tilde{A} e^{\rho}+\rho e^{\rho}\right) \leq \tilde{A}_{l}
\end{gathered}
$$

This is possible, for suitable $\tilde{A}, \tilde{A}_{l}$, if we take $C \tilde{A} e^{\rho} \leq \frac{\tilde{A}_{l}}{2}$ and $\tilde{A}_{l} e^{-\rho^{2}} \leq \frac{\tilde{A}}{2}$, i.e. $C e^{\rho-\rho^{2}} \leq \frac{1}{4}$.

For (4) with $m=2$, we have

$$
\left|\psi_{2}(\tau)\right| \leq\left(\sigma \tau^{-1}\right)^{\delta} \tau^{-2+\delta}+(\tau-\sigma) C A \tau^{-3} \leq \tau^{-2+\delta}
$$

for $\tau \geq \sigma$, large enough.

It thus remains to show that there exist $\psi_{m}\left(\tau_{0}\right) \in\left[-\tilde{A} \tau_{0}^{-2}, \tilde{A} \tau_{0}^{-2}\right], m=0,1$, such that (4) holds for all $\tau$. Suppose such $\psi_{m}\left(\tau_{0}\right)$ did not exist. Set $y=\tilde{A}^{-1} \tau_{0}^{2}\left(\psi_{0}\left(\tau_{0}\right), \psi_{1}\left(\tau_{0}\right)\right) \in$ $\mathcal{C}=[-1,1]^{2} \subseteq \mathbf{R}^{2}$ and $\phi=\tilde{A}^{-1} \tau^{2}\left(\psi_{0}(\tau), \psi_{1}(\tau)\right)$. We have shown that $\phi=\phi(\tau, y)$ is continuous in $\tau$ and $y$. Moreover, by the above assumption, for all $y$ there exists a first time $\tau(y)$, such that $\phi(\tau(y), y) \in \partial \mathcal{C}$. Also, by $(32)$, the flow $\phi(\tau, y)$ is transversal to $\partial \mathcal{C}$ (by induction, (32) holds up to time $\tau(y)$ ). This implies that $\tau(y)$ is continuous. Thus, $y \rightarrow \phi(\tau(y), y)$ is a continuous map from the unit square $\mathcal{C}$ in $\mathbf{R}^{2}$ to its boundary $\partial \mathcal{C}$, which is the identity on the boundary. Such a map can not exist, since $\mathcal{C}$ is contractible to a point and this map would then provide a homotopy between the identity map $S^{1} \rightarrow S^{1}$ and the constant map. Thus we can choose the $d_{0}, d_{1}$ such that (4), and hence all the other claims of the Proposition hold.

To summarize, the logic in the choice of constants is as follows: first, take $K$ in (2.57) large enough, and $\delta$ small enough, so that various estimates hold. For example, we shall use often the bound (see (2.7)): for $K$ in (2.57) large enough

$$
\int P(\xi)(1-\chi(\xi, \tau)) d \mu(\xi) \leq C(P) e^{-\tau}
$$

for any polynomial $P$, where $C(P)$ depends on $P$. This choice of $K$ and $\delta$ fixes the constants appearing in the bounds used in the proof. Then, we take $\rho$ large enough so that $(34,35)$ iterate $($ see $(36,37))$. Finally, take $\tau_{0}$ large, given $\rho$ and the various constants appearing in the proofs, so that we can write e.g. $\tau^{-\delta} \leq e^{-c \rho}$ or $C \leq \tau^{\delta}$ for $\tau \geq \tau_{0}$. In several estimates below, we replace $\sigma$ by $\tau$, which will be legitimate, using $\frac{\tau}{\sigma} \leq 2$.

We will now prove Lemmas 1-3.

Proof of Lemma 1. Let us denote $\tau-\sigma$ by $t$ and $K(\tau, \sigma)$ by $K_{t}$. $K_{t}$ is the fundamental solution of the linear equation $\dot{K}=(\mathcal{L}+V) K$ and we will use a Feynman-Kac representation for it. Since $\mathcal{L}$ is conjugated to the harmonic oscillator:

$$
e^{-\xi^{2} / 8} \mathcal{L} e^{\xi^{2} / 8}=\partial^{2}-\frac{\xi^{2}}{16^{2}}+\frac{1}{4}+1
$$


we may write

$$
K_{t}\left(\xi, \xi^{\prime}\right)=e^{t \mathcal{L}}\left(\xi, \xi^{\prime}\right) \int d \mu_{\xi \xi^{\prime}}^{t}(\omega) e^{\int^{t} V(\omega(s), \sigma+s) d s}
$$

where $d \mu_{\xi \xi^{\prime}}^{t}(\omega)$ is the oscillator measure on the continuous paths $\omega:[0, t] \rightarrow \mathbf{R}$ with $\omega(0)=\xi^{\prime}, \omega(t)=\xi$, i.e. the Gaussian probability measure with covariance kernel

$$
C\left(s, s^{\prime}\right)=\omega_{0}(s) \omega_{0}\left(s^{\prime}\right)+2\left(e^{-\frac{1}{2}\left|s-s^{\prime}\right|}-e^{-\frac{1}{2}\left|s+s^{\prime}\right|}+e^{-\frac{1}{2}\left|2 t-s^{\prime}+s\right|}-e^{-\frac{1}{2}\left|2 t-s^{\prime}-s\right|}\right),
$$

and mean $\int d \mu_{\xi \xi^{\prime}}^{t}(\omega) \omega(s)=\omega_{0}(s)$, where

$$
\omega_{0}(s)=\left(\sinh \frac{t}{2}\right)^{-1}\left(\xi \sinh \frac{s}{2}+\xi^{\prime} \sinh \frac{t-s}{2}\right) .
$$

The kernel of $e^{t \mathcal{L}}$ is given explicitely by Mehler's formula 14

$$
e^{t \mathcal{L}}\left(\xi, \xi^{\prime}\right)=\left[4 \pi\left(1-e^{-t}\right)\right]^{-1 / 2} e^{t} \exp \left[-\frac{\left(\xi e^{-t / 2}-\xi^{\prime}\right)^{2}}{4\left(1-e^{-t}\right)}\right] .
$$

Although the proof of Lemma 1 is long, most of it can be understood easily by considering (41), (44). If we replace $K_{t}$ by $e^{t \mathcal{L}}$, we understand the LHS of (10), coming from (2.11). But the potential $V$, see (2.42), is of order $\tau^{-1}$ for $\xi$ of order one. The precise estimate is done in Lemma 5 below and gives as a correction the RHS of (10). In (11), the term on the LHS comes from $V$, as we saw in (2.52), and the RHS is as in (10). For (12), the first term on the RHS comes from the fact that $e^{t \mathcal{L}}$ contracts $\theta^{\perp}$, which follows also from (2.11); however, we shall use an integration by parts and the explicit formula (44), in order not to expand as in (2.54). The second term in the RHS of (12) is the contribution to small $\xi$, coming from large $\xi^{\prime}$. Looking at (44), we see that this contribution is small for large $t$. Finally, in (13), the first term in the RHS, i.e. the contribution from large $\xi^{\prime}$, is suppressed because the potential is no longer small, while the one coming from small $\xi^{\prime}$ is controlled because $\psi_{s}$ is bounded by $\mathcal{O}\left(\tau^{-1 / 2}\right)$.

Let us now bound each term in (8). Consider first $\theta_{m}$ : let $k_{m}=h_{m}\left\|h_{m}\right\|^{-2}$. Then $\theta_{m}(\tau)=\left(k_{m}, \chi_{\tau} K_{t} \psi(\sigma)\right)$, where $\chi_{\tau}=\chi(\xi, \tau)$, and $\psi_{m}(\sigma)=\left(k_{m}, \chi_{\sigma} \psi(\sigma)\right)$. We write for $m=0,1$,

$$
\chi_{\tau} K_{t}-e^{\left(1-\frac{m}{2}\right) t} \chi_{\sigma}=\chi_{\tau}\left(e^{t \mathcal{L}}-e^{\left(1-\frac{m}{2}\right) t}\right)+\chi_{\tau}\left(K_{t}-e^{t \mathcal{L}}\right)+e^{\left(1-\frac{m}{2}\right) t}\left(\chi_{\tau}-\chi_{\sigma}\right) .
$$

Consider the first term in (45). Using $(2,3)$ and $(2.11)$, we have, writing $\chi=\chi_{\tau}$, $\psi=\psi(\sigma)$,

$$
\begin{aligned}
\left(k_{m}, \chi\left(e^{t \mathcal{L}}-e^{\left(1-\frac{m}{2}\right) t}\right) \psi\right) & =\sum_{r=0}^{2}\left[\left(e^{\left(1-\frac{r}{2}\right) t}-e^{\left(1-\frac{m}{2}\right) t}\right) \psi_{r}\left(k_{m}, \chi h_{r}\right)\right] \\
& +\left(k_{m}, \chi\left(e^{t \mathcal{L}}-e^{\left(1-\frac{m}{2}\right) t}\right) \psi^{\perp}\right)+\left(k_{m}, \chi\left(e^{t \mathcal{L}}-e^{\left(1-\frac{m}{2}\right) t}\right) \psi_{l}\right)
\end{aligned}
$$

For the first term in (46), use (39) to get

$$
\left|\left(k_{m}, \chi h_{r}\right)-\delta_{m r}\right| \leq C e^{-\tau} .
$$


Indeed, by definition, $\left(k_{m}, h_{r}\right)=\delta_{m r}$.

For the two other terms and for later purposes, we need the following property of the kernel of $e^{t \mathcal{L}}$, that follows easily from the explicit expression (44):

Lemma 4. Let $\left|\psi\left(\xi^{\prime}\right)\right| \leq\left(1+\left|\xi^{\prime}\right|\right)^{p}$, for $p \geq 0$. Then

$$
\left|\left(e^{t \mathcal{L}} \psi\right)(\xi)\right| \leq C e^{t}\left(1+e^{-t / 2}|\xi|\right)^{p}
$$

With Lemma 4 and equations $(5,39)$, we get, since $\left(k_{m},\left(e^{t \mathcal{L}}-e^{\left(1-\frac{m}{2}\right) t}\right) \psi^{\perp}\right)=0$, for $m \leq 2$,

$$
\left|\left(k_{m}, \chi\left(e^{t \mathcal{L}}-e^{\left(1-\frac{m}{2}\right) t}\right) \psi^{\perp}\right)\right|=\left|\left(k_{m},(1-\chi)\left(\left(e^{t \mathcal{L}}-1\right)-\left(e^{\left(1-\frac{m}{2}\right) t}-1\right)\right) \psi^{\perp}\right)\right| \leq C A t e^{t} e^{-\tau}
$$

Indeed, we may write $e^{t \mathcal{L}}-1=\int_{0}^{t} d s \mathcal{L} e^{s \mathcal{L}}$, and use the fact that $(1-\chi) k_{m} \in \mathcal{D}(\mathcal{L})$ and $\mathcal{L}(1-\chi) k_{m}$ has support in $|\xi| \geq K$, which follows from the smoothness of $\chi$.

Finally, for the last term in (46), using (6), and reasoning as above,

$$
\begin{aligned}
& \left|\left(k_{m}, \chi\left(e^{t \mathcal{L}}-1\right) \psi_{l}\right)\right| \leq A_{l} \sigma^{-\frac{1}{2}} \int d \mu(\xi) d \xi^{\prime}\left|\mathcal{L}\left(k_{m} \chi_{\tau}\right)(\xi)\right| \\
& \cdot \int_{0}^{t} d s e^{s \mathcal{L}}\left(\xi, \xi^{\prime}\right)\left(1-\chi\left(\xi^{\prime}, \sigma\right)\right) \leq C t e^{-\tau} .
\end{aligned}
$$

and a similar bound for the term with $1-e^{\left(1-\frac{m}{2}\right) t}$. Indeed, if we insert (44) and (2.7) into (50), we end up with the estimate

$$
\sup _{\xi \leq 2 K \tau^{1 / 2}, \xi^{\prime} \geq K \sigma^{1 / 2}} e^{-\frac{\xi^{2}}{4}-\frac{\left(\xi e^{-s / 2}-\xi^{\prime}\right)^{2}}{4\left(1-e^{-s}\right)}} \leq e^{-2 \tau} .
$$

for $K$ large enough. We may use the square root in the LHS of (51) to control the integrals in (50) and the factor $e^{t}$ in (48). The constant $A_{l}$ in (50) is bounded by the factor $\sigma^{-\frac{1}{2}}$, for $\sigma$ large enough.

For the second term in (45), we write again

$$
\left(k_{m}, \chi\left(K_{t}-e^{t \mathcal{L}}\right) \psi\right)=\sum_{r=0}^{2}\left(k_{m}, \chi\left(K_{t}-e^{t \mathcal{L}}\right) h_{r}\right) \psi_{r}+\left(k_{m}, \chi\left(K_{t}-e^{t \mathcal{L}}\right)\left(\psi^{\perp}+\psi_{l}\right)\right) .
$$

Now we need some properties of $K_{t}$ :

Lemma 5. The kernel $K_{t}\left(\xi, \xi^{\prime}\right)$ given by (41)satisfies

$$
K_{t}\left(\xi, \xi^{\prime}\right)=e^{t \mathcal{L}}\left(\xi, \xi^{\prime}\right)\left(1+\frac{1}{\tau} P_{2}\left(\xi, \xi^{\prime}\right)+R\left(\xi, \xi^{\prime}\right)\right)
$$

where $P_{2}$ is a polynomial

$$
P_{2}\left(\xi, \xi^{\prime}\right)=\sum_{m+n \leq 2} p_{m n} \xi^{m} \xi^{\prime n}
$$


with $\left|p_{m n}\right|<C t$, and $R$ is bounded by

$$
\left|R\left(\xi, \xi^{\prime}\right)\right| \leq C t(1+t) \tau^{-2}\left(1+|\xi|+\left|\xi^{\prime}\right|\right)^{4}
$$

Moreover,

$$
\left|\left(k_{2},\left(K_{t}-\left(\frac{\sigma}{\tau}\right)^{2}\right) h_{2}\right)\right| \leq C t(1+t) \tau^{-2}
$$

Using (53)-(55),(4), Lemma 4 and (2.7), we have, for $m \leq 2$,

$$
\begin{aligned}
& \left|\left(k_{m}, \chi\left(K_{t}-e^{t \mathcal{L}}\right) h_{r}\right) \psi_{r}\right| \leq C A t \tau^{-3} \quad r=0,1 \\
& \left|\left(k_{m}, \chi\left(K_{t}-e^{t \mathcal{L}}\right) h_{2}\right) \psi_{2}\right| \leq C t \tau^{-3+\delta} .
\end{aligned}
$$

By (5) and Lemma 4 , the $\psi^{\perp-t e r m}$ in (52) also satisfies a bound like the first inequality in (57) and the $\psi_{l}$-term is bounded as in (50). For the last term in (45), involving $\chi_{\tau}-\chi_{\sigma}$, we can bound its contribution by $C t e^{-\tau}$, using (2.7) as in (39). Hence, combining $(45,47,49,50,57)$, we get

$$
\left|\theta_{m}-e^{\left(1-\frac{m}{2}\right) t} \psi_{m}\right| \leq C t \tau^{-3+\delta}, \quad m=0,1
$$

For $m=2$, we write first

$$
\chi_{\tau} K_{t}-\left(\frac{\sigma}{\tau}\right)^{2} \chi_{\sigma}=\chi_{\tau}\left(K_{t}-\left(\frac{\sigma}{\tau}\right)^{2}\right)+\left(\chi_{\tau}-\chi_{\sigma}\right)\left(\frac{\sigma}{\tau}\right)^{2}
$$

Then, combine the previous bounds with (56), using only the first inequality in (57) since we use (56) for the $r=2$ term. We get:

$$
\left|\theta_{2}-\left(\frac{\sigma}{\tau}\right)^{2} \psi_{2}\right| \leq C A t \tau^{-3} .
$$

This proves $(10,11)$.

Next, consider $\theta^{\perp}$ in (8). Let $P^{\perp}$ be the projection in $L^{2}(\mathbf{R}, d \mu)$ on the corresponding subspace. We write, using $(2,3)$,

$$
\theta^{\perp}=P^{\perp} \chi K_{t} \psi=P^{\perp} \chi K_{t} \psi^{\perp}+\sum_{r=0}^{2} \psi_{r} P^{\perp} \chi K_{t} h_{r}+P^{\perp} \chi K_{t} \psi_{l}
$$

and consider again the various terms separately.

For the first term, we can write

$$
\left(K_{t} \psi^{\perp}\right)=\int d \xi^{\prime} M\left(\cdot, \xi^{\prime}\right) f\left(\xi^{\prime}\right)
$$

where,

$$
M\left(\xi, \xi^{\prime}\right)=e^{\xi^{\prime 2} / 4} K_{t}\left(\xi, \xi^{\prime}\right), \quad f\left(\xi^{\prime}\right)=e^{-\xi^{\prime 2} / 4} \psi^{\perp}\left(\xi^{\prime}, \sigma\right)
$$

i.e., see $(41,44)$,

$$
\begin{aligned}
M\left(\xi, \xi^{\prime}\right) & =\left[4 \pi\left(1-e^{-t}\right)\right]^{-1 / 2} e^{t} e^{\xi^{2} / 4} e^{-\frac{\left(\xi-e^{-t / 2} \xi^{\prime}\right)^{2}}{4\left(1-e^{-t}\right)}}\left\langle e^{V}\right\rangle\left(\xi, \xi^{\prime}\right) \\
& \equiv N\left(\xi, \xi^{\prime}\right)\left\langle e^{V}\right\rangle\left(\xi, \xi^{\prime}\right)
\end{aligned}
$$


where we used the identity

$$
\frac{\left(\xi e^{-t / 2}-\xi^{\prime}\right)^{2}}{1-e^{-t}}-\xi^{\prime 2}=-\xi^{2}+\frac{\left(\xi-e^{-t / 2} \xi^{\prime}\right)^{2}}{1-e^{-t}}
$$

and the notation

$$
\left\langle e^{V}\right\rangle\left(\xi, \xi^{\prime}\right)=\int d \mu_{\xi \xi^{\prime}}^{t}(\omega) e^{\int^{t} V(\omega(s), \sigma+s) d s}
$$

Now, $\left(\psi^{\perp}, h_{m}\right)=0, m \leq 2$ means (see $\left.(2.7)\right) \int f\left(\xi^{\prime}\right) \xi^{\prime m} d \xi^{\prime}=0, m \leq 2$. Thus, let $f^{(-m)}$ be the $m$ :th antiderivative of $f$, i.e.

$$
f^{(-m-1)}(\xi)=\int_{-\infty}^{\xi} d \xi^{\prime} f^{(-m)}\left(\xi^{\prime}\right) .
$$

We have

Lemma 6. For $f$ defined in (62),

$$
\left|f^{(-m)}(\xi)\right| \leq C A \tau^{-2}(1+|\xi|)^{3-m} e^{-\xi^{2} / 4}
$$

for $m \leq 3$

Now write (61) by integrating by parts,

$$
\begin{aligned}
\left(K_{t} \psi^{\perp}\right)(\xi)= & \sum_{r=0}^{2} \int \partial_{\xi^{\prime}}^{r} N\left(\xi, \xi^{\prime}\right) \partial_{\xi^{\prime}}\left\langle e^{V}\right\rangle\left(\xi, \xi^{\prime}\right) f^{(-r-1)}\left(\xi^{\prime}\right) d \xi^{\prime} \\
& +\int \partial_{\xi^{\prime}}^{3} N\left(\xi, \xi^{\prime}\right)\left\langle e^{V}\right\rangle\left(\xi, \xi^{\prime}\right) f^{(-3)}\left(\xi^{\prime}\right) d \xi^{\prime}
\end{aligned}
$$

We need the integration by parts formula for Gaussian measures [6]:

$$
\begin{aligned}
\partial_{\xi^{\prime}}\left\langle e^{V}\right\rangle\left(\xi, \xi^{\prime}\right)= & \frac{1}{2} \int_{0}^{t} d s d s^{\prime} \partial_{\xi^{\prime}} C\left(s, s^{\prime}\right) \int d \mu_{\xi \xi^{\prime}}^{t}(\omega) V^{\prime}(\omega(s), \sigma+s) V^{\prime}\left(\omega\left(s^{\prime}\right), \sigma+s^{\prime}\right) e^{\int V} \\
& +\frac{1}{2} \int_{0}^{t} d s \partial_{\xi^{\prime}} C(s, s) \int d \mu_{\xi \xi^{\prime}}^{t}(\omega) V^{\prime \prime}(\omega(s), \sigma+s) e^{\int V}
\end{aligned}
$$

Since (see (2.42))

$$
\begin{aligned}
V & \leq \frac{C}{\tau} \\
\left|\frac{d^{n} V}{d \xi^{n}}\right| & \leq C \tau^{-n / 2}, n=0,1,2
\end{aligned}
$$

and $C\left(s, s^{\prime}\right)$ is given by (42), we have $\int d \mu_{\xi \xi^{\prime}}^{t}(\omega) e^{\int V} \leq C$ and

$$
\left|\partial_{\xi^{\prime}}\left\langle e^{V}\right\rangle\left(\xi, \xi^{\prime}\right)\right| \leq C \tau^{-1} t(1+t)\left(|\xi|+\left|\xi^{\prime}\right|\right) .
$$


As for $\partial_{\xi^{\prime}}^{r} N$, we get from (63), for $t>1$,

$$
\left|\partial_{\xi^{\prime}}^{r} N\left(\xi, \xi^{\prime}\right)\right| \leq C e^{-\frac{r t}{2}}\left(|\xi|+\left|\xi^{\prime}\right|\right)^{r} e^{\xi^{2} / 4} e^{t \mathcal{L}}\left(\xi, \xi^{\prime}\right) .
$$

where we used $(64,44)$ to rewrite the RHS. Thus, from (66), (67), (70), (71) and Lemma 4 , using $\tau^{-1} t(1+t) \leq e^{\frac{-3 t}{2}}$ for $\tau$ large, we get,

$$
\left|\left(K_{t} \psi^{\perp}\right)(\xi)\right| \leq C A \tau^{-2} e^{-t / 2}(1+|\xi|)^{3} .
$$

To control $P^{\perp} \chi K_{t} \psi^{\perp}$, we use the following remark; let $X(\xi)$ satisfy

$$
|X(\xi)| \leq \eta(1+|\xi|)^{3}
$$

Then, using (2.7), we have

$$
\left|\left(k_{m}, X\right)\right| \leq C \eta
$$

Hence, $P^{\perp} X(\xi)=X(\xi)-\sum_{m=0}^{2}\left(k_{m}, X\right) h_{m}(\xi)$ satisfies

$$
\left|P^{\perp} X(\xi)\right| \leq C \eta(1+|\xi|)^{3}
$$

So, $P^{\perp} \chi K_{t} \psi^{\perp}$ satisfies a bound like (72). If $t \leq 1$, since the derivatives in (71) bring extra factors of $t^{-1}$, so we do not integrate by parts as in (67), but derive the bound (12) for that term in (60) directly from Lemma 5, Lemma 4 and (5).

Now consider the second term in (60). Since $K_{t}$ is given by Lemma 5 , we obtain

$$
\left|\psi_{r}\left(\chi K_{t} h_{r}\right)(\xi)-\psi_{r} e^{t\left(1-\frac{r}{2}\right)}\left(\chi h_{r}\right)(\xi)\right| \leq C A \tau^{-3+\delta+1 / 2} e^{t}\left(1+|\xi|^{3}\right) .
$$

Indeed, we get $A \tau^{-2+\delta}$ from (4); for the $P_{2}$ term in (53), we have $\tau^{-1}$ and, using Lemma 4 ,

$$
\left|e^{t \mathcal{L}}\left(P_{2} h_{r}\right)(\xi)\right| \leq C e^{t}\left(1+|\xi|^{4}\right)
$$

since $r \leq 2$. But, on the support of $\chi,|\xi| \leq 2 K \tau^{1 / 2}$, so we can replace one power of $|\xi|$ by $2 K \tau^{1 / 2}$. Similarly, for $R$ in (53), we get from (55) and Lemma 4, a bound with $\tau^{-2}$ and $(1+|\xi|)^{6}$ and we control $|\xi|^{3}$ by $\tau^{3 / 2}$. Now, using (73), we get a bound like (74) on $P^{\perp}\left(\psi_{r}\left(\chi K_{t} h_{r}\right)(\xi)-\psi_{r} e^{t\left(1-\frac{r}{2}\right)}\left(\chi h_{r}\right)(\xi)\right)$. We still have to consider $\psi_{r} e^{t\left(1-\frac{r}{2}\right)} P^{\perp} \chi h_{r}, r \leq 2$. But, by definition, $P^{\perp} h_{r}=0$, and we can replace $\chi$ by $(1-\chi)$, and use

$$
\left|(1-\chi) h_{r}\right| \leq \tau^{-1 / 2}(1+|\xi|)^{3}
$$

since $r \leq 2$ and $|\xi| \geq K \tau^{1 / 2}$ on the support of $(1-\chi)$. Then, by (73) again, $P^{\perp}(1-\chi) h_{r}$ satisfies a similar bound. Hence, the second term of (60) has a bound like the RHS of (74).

For the last term in (60), we use the bound (69) on $V$, to get From $(41), K_{t}\left(\xi, \xi^{\prime}\right) \leq$ $C e^{t \mathcal{L}}\left(\xi, \xi^{\prime}\right)$. Then, using (6) and (44), we have

$$
\begin{aligned}
& \left\|\left(1+|\xi|^{3}\right)^{-1} \chi K_{t} \psi_{l}\right\|_{\infty} \leq C A_{l} e^{t} \tau^{-1 / 2} \sup _{\xi, \xi^{\prime}}\left(1+|\xi|^{3}\right)^{-1} \\
& \cdot e^{-c\left(\xi e^{-t / 2}-\xi^{\prime}\right)^{2}} \chi(\xi, \sigma+t)\left(1-\chi\left(\xi^{\prime}, \sigma\right)\right) \leq \begin{cases}C A_{l} \tau^{-2} & t \leq t_{0} \\
e^{-\tau} & t>t_{0}\end{cases}
\end{aligned}
$$


for a suitable $t_{0}$. Indeed, for $t$ large, we get $e^{-2 \tau} \leq e^{-\tau}\left(C A_{l} e^{t}\right)^{-1}$ from $e^{-c\left(\xi e^{-t / 2}-\xi^{\prime}\right)^{2}}$ and the characteristic functions, while, for $t$ small, we get $\xi \simeq \xi^{\prime}$ and therefore $\left(1+|\xi|^{3}\right)^{-1} \leq$ $c \tau^{-3 / 2}$ from $\left(1-\chi\left(\xi^{\prime}, \sigma\right)\right)$. Then, proceeding as for $(72,73)$, we get a bound on the last term of (60), which can be written as $C A_{l} e^{-t^{2}} \tau^{-2}\left(1+|\xi|^{3}\right)$ for $\tau$ large. This bound is of course rather arbitrary, but convenient.

Hence, combining (72),(74) and (75), and using $\tau^{-1 / 2+\delta} e^{t} \leq e^{-t / 2}$ in (74), we have

$$
\left|\theta^{\perp}(\xi, \tau)\right| \leq C \tau^{-2}\left(A e^{-t / 2}+A_{l} e^{-t^{2}}\right)\left(1+|\xi|^{3}\right) .
$$

This proves (12).

Only $\theta_{l}$ remains to be bounded. We have (see $(9)$ ),

$$
\theta_{l}=(1-\chi) K_{t} \psi=(1-\chi) K_{t}\left(\psi_{s}+\psi_{l}\right)
$$

By $(3,4,5)$, we have, using $\chi|\xi| \leq 2 K \sigma^{1 / 2},\left|\psi_{s}\right| \leq C A \sigma^{-1 / 2}$. Now, use $K_{t} \leq C e^{t \mathcal{L}}$ (from (69) and (41)) and (48) with $p=0$, to get

$$
\left\|K_{t} \psi_{s}\right\| \leq C A e^{t} \tau^{-\frac{1}{2}}
$$

This gives a bound on the first term of (77).

For the second term, we use

Lemma 7. Let $\chi$ be the function (2.57). Then,

$$
\left\|K_{t}(1-\chi)\right\|_{\infty} \leq C e^{-t / p}
$$

Thus,

$$
\left\|K_{t} \psi_{l}\right\|_{\infty} \leq C A_{l} e^{-t / p} \tau^{-1 / 2}
$$

The bound (13) for $\theta_{l}$ follows from (78) and (80).

Now, we shall prove Lemmas 5-7 that were used in the proof of Lemma 1.

Proof of Lemma 5. We start with the Feynman-Kac formula (41). Let

$$
M(\lambda)=\int d \mu_{\xi \xi^{\prime}}^{t}(\omega) e^{\lambda \int_{0}^{t} V(\omega(s), \sigma+s) d s} .
$$

$M$ is $C^{2}$ in $\lambda\left(\right.$ in fact $\left.C^{\infty}\right)$ and

$$
M(1)=1+\int_{0}^{t} d s \int d \mu_{\xi \xi^{\prime}}^{t}(\omega) V(\omega(s), \sigma+s)+\tilde{M}
$$

with

$$
\tilde{M}=\int_{0}^{1} d \lambda(1-\lambda) \int_{0}^{t} d s d s^{\prime} \int d \mu_{\xi \xi^{\prime}}^{t}(\omega) V(\omega(s), \sigma+s) V\left(\omega\left(s^{\prime}\right), \sigma+s^{\prime}\right) e^{\lambda \int_{0}^{t} V(\omega(u), \sigma+u) d u} .
$$


We have the following bounds for $V$ given by (2.42):

$$
\begin{aligned}
V & \leq \frac{C}{\tau} \\
|V(\omega, \tau)| & \leq C \tau^{-1}(1+|\omega|)^{2} \\
V(\omega, \tau) & =\tau^{-1} Q(\omega)+\tilde{V}(\omega, \tau)
\end{aligned}
$$

with $Q(\omega)$ a polynomial of degree 2 in $\omega$ with bounded coefficients and where $\tilde{V}$ satisfies

$$
|\tilde{V}(\omega, \tau)| \leq \frac{C}{\tau^{2}}(1+|\omega|)^{4}
$$

Insert now (86) in (82) and, in (83), insert (84) in the exponent and (85) elsewhere. Using formulas (42), (43) for the covariance of $\mu$, the claims (53)-(55) follow. $P$ is produced by $Q$ in (86), while $R$ collects $\tilde{M}$ and $\tilde{V}$.

Finally, we prove estimate (56). We want to show that the contribution of the second term in $(82)$ to $\left(k_{2}, K_{t} h_{2}\right)$ is as claimed in (56).

Note that the term $\tilde{M}$ in (82) can be absorbed into the RHS of (56). Also, by (86), we may replace $V$ in (82) by $\tau^{-1} Q$, the error again being absorbed into the RHS of (56).

Using (14) and (2.42), we compute

$$
\tau^{-1} Q(\omega)=-\frac{p b}{\tau(p-1)^{2}} h_{2}(\omega)=\frac{-h_{2}(\omega)}{4 \tau}
$$

We could now calculate the Gaussian integral in (82) directly, using the covariance (42) and the fact that $Q$ is a polynomial. The result, however, can be obtained directly, by noting that the above estimates imply that

$$
\left|\frac{d}{d \tau}\left(k_{2}, K_{t} h_{2}\right)-\frac{d}{d \tau} \psi_{2}^{(0)}(\tau)\right| \leq C(1+t) \tau^{-2}
$$

(with $t=\tau-\sigma$ ) where $\psi_{2}^{(0)}$ solves the equation

$$
\frac{d}{d \tau} \psi_{2}^{(0)}=\left(k_{2}, \tau^{-1} Q(\cdot) h_{2}\right) \psi_{2}^{(0)}=-\frac{\left(h_{2}^{2}, h_{2}\right)}{4 \tau\left\|h_{2}\right\|^{2}} \psi_{2}^{(0)}=-2 \tau^{-1} \psi_{2}^{(0)}
$$

with initial condition $\psi_{2}^{(0)}(\sigma)=1$. Indeed, we may first replace, with error bounded by (89), $K_{t}$ by $e^{t \mathcal{L}}\left(1+\int_{0}^{t} d s \frac{\langle Q(\omega(s))\rangle\left(\xi, \xi^{\prime}\right)}{\sigma+s}\right)$ (use (82-87)). Then, using $e^{t \mathcal{L}} k_{2}=k_{2}$, we replace $\left(k_{2}, K_{t} h_{2}\right)$ by

$$
e^{\left(k_{2}(\cdot), \int_{0}^{t} d s \frac{\langle Q(\omega(s))\rangle(\cdot, \cdot)}{\sigma+s} h_{2}(\cdot)\right)}
$$

where, again, the error is bounded by (89). Differentiating gives (89, 90). The solution of $(90)$ is $\psi_{2}^{(0)}(\tau)=\left(\frac{\sigma}{\tau}\right)^{2}$, which yields $(56)$.

Proof of Lemma 6. We show: let $\int f d x=0$ and $|f(x)| \leq A\left(1+|x|^{p}\right) e^{-x^{2} / 4}$ then

$$
\left|f^{(-1)}(x)\right| \leq C A\left(1+|x|^{p-1}\right) e^{-x^{2} / 4} .
$$


The claim then follows by induction in $m$ using the fact that $\int f(x) x^{m} d x=0$ for $m \leq 2$ implies $\int f^{(-m)}(x) d x=0$ for $m \leq 2$; (91) follows from a simple calculation: let e.g. $x>0$. Then, since $\int f d x=0$,

$$
\begin{aligned}
& \left|f^{(-1)}(x)\right| \leq A \int_{x}^{\infty}\left(1+|y|^{p}\right) e^{-y^{2} / 4} d y \leq \\
& A \int_{x^{2} / 4}^{\infty} u^{-\frac{1}{2}}\left(1+|u|^{p / 2}\right) e^{-u} d u \leq C A\left(1+|x|^{p-1}\right) e^{-x^{2} / 4}
\end{aligned}
$$

Proof of Lemma 7. We will use both the oscillator formula (41) and another FeynmanKac formula for $K_{t}$, in terms of the Wiener measure, which follows from the conjugation (40):

$$
K_{t}\left(\xi, \xi^{\prime}\right)=\exp \left[\left(1+\frac{1}{4}\right) t+\frac{1}{8}\left(\xi^{2}-\xi^{\prime 2}\right)\right] \int d \nu_{\xi \xi^{\prime}}^{t}(\omega) e^{U(\omega)}
$$

where

$$
U(\omega)=\int_{0}^{t}\left(V(\omega(t), \sigma+t)-\frac{1}{16} \omega(t)^{2}\right) d t
$$

and $d \nu_{\xi \xi^{\prime}}^{t}$ is the Wiener measure on continuous paths $\omega:[0, t] \rightarrow \mathbf{R}, \omega(0)=\xi^{\prime}, \omega(t)=\xi$ with the normalization

$$
\int d \nu_{\xi \xi^{\prime}}^{t}(\omega)=e^{t \partial^{2}}\left(\xi, \xi^{\prime}\right)
$$

We want to estimate

$$
\int K_{t}\left(\xi, \xi^{\prime}\right)\left(1-\chi\left(\xi^{\prime}, \sigma\right)\right) d \xi^{\prime} \leq C e^{-t / p}
$$

uniformly in $\xi$. Let us divide the integral into two regions:

(a) Let $\left|\xi e^{-t / 2}-\xi^{\prime}\right| \geq\left|\xi^{\prime}\right| / 4$. By (84), (41) and (44)

$$
K_{t}\left(\xi, \xi^{\prime}\right) \leq C\left(1-e^{-t}\right)^{-\frac{1}{2}} e^{t} e^{-\frac{c \xi^{\prime 2}}{1-e^{-t}}}
$$

and that contribution to (95) is bounded by $e^{-\sigma}$ for $K$ in (2.57) (and $\sigma$ ) large enough. (b) Let $\left|\xi e^{-t / 2}-\xi^{\prime}\right| \leq \frac{1}{4}\left|\xi^{\prime}\right|$, hence, for $\xi^{\prime}>0$, say, $\xi \in\left[\frac{3}{4} e^{t / 2} \xi^{\prime}, \frac{5}{4} e^{t / 2} \xi^{\prime}\right]$. we use the representation (92) and condition on the first time $t$ such that $\omega(t)=\frac{1}{2} \xi^{\prime}$, if $\omega$ visits $\frac{1}{2} \xi^{\prime}$ at all. So,

$$
\begin{aligned}
\int d \nu_{\xi, \xi^{\prime}}^{t}(\omega) e^{U(\omega)} & =\int_{0}^{t} d t_{1} \int d \nu_{\frac{1}{2} \xi^{\prime}, \xi^{\prime}}^{t_{1}}\left(\omega_{1}>\frac{\xi^{\prime}}{2}\right) \int d \nu_{\xi, \frac{1}{2} \xi^{\prime}}^{t-t_{1}}\left(\omega_{2}\right) e^{U\left(\omega_{1} \cup \omega_{2}\right)} \\
& +\int d \nu_{\xi \xi^{\prime}}^{t}(\omega) \chi\left(\omega>\frac{1}{2} \xi^{\prime}\right) e^{U(\omega)}
\end{aligned}
$$

where $d \nu_{a, b}^{t}(\omega>a)$ is the measure on paths $\omega$ such that $\omega(0)=b, \omega(t)=a$, and $\omega(s)>a$, for $s<t$, defined by

$$
\int F(\omega) d \nu_{a, b}^{t}(\omega>a)=\left.2 \frac{d}{d x} \int F(\omega) d \nu_{x, b}^{t}(\omega)\right|_{x=a}
$$


One can check that this defines an expectation and that (97) holds by the method of images. Below we shall only use the formula

$$
\int d \nu_{a, b}^{t}(\omega>a)=\frac{(b-a)}{\left(4 \pi t^{3}\right)^{\frac{1}{2}}} e^{-\frac{(a-b)^{2}}{4 t}}
$$

which is the probability density that $t$ is the first time at which $\omega$, starting from $b$, reaches $a$. Hence,

$$
\int_{0}^{t} d t_{1} \int d \nu_{\frac{1}{2} \xi^{\prime}, \xi^{\prime}}^{t_{1}}\left(\omega_{1}>\frac{\xi^{\prime}}{2}\right) \leq 1
$$

For the second term in (97) (this is where the extra contraction in large $\xi$ comes from!), since $|\omega(t)| \geq \frac{K}{2} \tau^{1 / 2}$ (because of the characteristic function in (95)), we have from (93, $2.42)$

$$
\begin{aligned}
U(\omega) & \leq\left[-\frac{p}{p-1}+p\left(\left(\frac{c}{K}\right)^{\frac{1}{p-1}}+\frac{a}{\tau}\right)^{p-1}\right] t-\frac{1}{16} \int_{0}^{t} \omega(t)^{2} d t \\
& \leq-\left(1+\frac{1}{p}\right) t-\frac{1}{16} \int_{0}^{t} \omega(t)^{2} d t
\end{aligned}
$$

for $K$ (and $\tau$ ) large enough. Using $(92,41)$, the contribution of the second term in $(97)$ to $(95)$ (see $(92))$ is then bounded by

$$
e^{-\left(1+\frac{1}{p}\right) t} \int e^{t \mathcal{L}}\left(\xi, \xi^{\prime}\right) d \xi^{\prime} \leq e^{-t / p}
$$

since $\mathcal{L} 1=1$. For the first term in (97), use (84) to bound

$$
U\left(\omega_{1} \cup \omega_{2}\right) \leq-\frac{1}{16} \int_{0}^{t-t_{1}} \omega_{2}(t)^{2} d t+\frac{C t}{\tau}
$$

so that, using (98), this term is bounded by

$$
C \sup _{t_{1} \in[0, t]}\left(e^{\left(t-t_{1}\right)\left(\partial^{2}-\frac{\xi^{2}}{16}\right)}\left(\xi, \frac{\xi^{\prime}}{2}\right)\right) \cdot
$$

Hence, using $(40,92)$ its contribution to $K_{t}$ is bounded by

$$
C \sup _{t_{1} \in[0, t]} e^{\left(t-t_{1}\right) \mathcal{L}}\left(\xi, \frac{\xi^{\prime}}{2}\right) .
$$

From the Mehler formula (44), since (let $\zeta=t-t_{1}$ )

$$
\left(\xi e^{-\zeta / 2}-\frac{\xi^{\prime}}{2}\right)^{2} \geq\left(\frac{\xi^{\prime}}{4}\right)^{2}
$$

(recall that $\left.\xi \geq \frac{3}{4} e^{t / 2} \xi^{\prime}\right)$, this term, inserted in $(95)$, contributes $\mathcal{O}\left(e^{-c \xi^{\prime 2}}\right)=\mathcal{O}\left(e^{-\sigma}\right)$. Thus the claim follows by combining this bound with $(96,99)$. 
Proof of Lemma 2. We shall prove the following bounds for $\alpha$ defined in (2.44):

$$
\begin{aligned}
\left|\alpha_{m}(s)\right| & \leq C s^{-2} m=0,1 \\
\left|\alpha_{2}(s)\right| & \leq C s^{-3} \\
\left|\alpha^{\perp}(\xi, s)\right| & \leq C\left(1+|\xi|^{3}\right) s^{-2} \\
\left\|\alpha_{l}(\cdot, s)\right\|_{\infty} & \leq C s^{-\frac{1}{2}}
\end{aligned}
$$

Then, using Lemma 1 with $\psi(\sigma)$ replaced by $\alpha(s), K(\tau, \sigma)$ replaced by $K(\tau, s)$, and integrating over $s$, we get (16-19). We shall first show that, when $a=2 b(p-1)^{\frac{2 p-1}{1-p}}$, (104) holds and then show that, if we choose $b=b^{*},(105)$ holds also. For (104), we note that, using (2.7)

$$
\left|\left(h_{m},-\dot{\varphi}_{b}+W \eta_{0}-\dot{\eta}_{0}+M\left(\eta_{0}\right)\right)\right| \leq C s^{-2}
$$

Indeed, we may Taylor-expand $M\left(\eta_{0}\right)$ in the scalar product. From the expression (2.34) for $\varphi_{b}$, we deduce (we set $\left.c_{p}=(p-1)^{\frac{1}{1-p}}\right)$

$$
\left|\left(h_{m}, \varphi_{b}^{\prime \prime}+c_{p} \frac{2 b}{s(p-1)^{2}}\right)\right| \leq C s^{-2}
$$

but $\frac{c_{p}}{(p-1)^{2}}=(p-1)^{\frac{2 p-1}{1-p}}$ and therefore, the contribution of $\varphi_{b}^{\prime \prime}$ to order $s^{-1}$ and of $\eta_{0}=\frac{a}{s}$ in (2.44) cancel each other (actually, in (109) only $m=0$ is needed, since $h_{1}$ is orthogonal to constants). This proves (104).

Next, consider (105). Since $h_{2}$ is orthogonal to constants, $\left(\eta_{0}, h_{2}\right)=\left(\dot{\eta}_{0}, h_{2}\right)=0$, and we want to show that

$$
\left|\left(h_{2}, \varphi_{b}^{\prime \prime}-\dot{\varphi}_{b}+W \eta_{0}+M\left(\eta_{0}\right)\right)\right| \leq C s^{-3}
$$

Again we Taylor-expand and get

$$
\begin{aligned}
& \left|\left(h_{2}, \varphi_{b}^{\prime \prime}-\dot{\varphi}_{b}-c_{p}\left(\frac{p b^{2}}{2(p-1)^{4}} \partial^{2} \xi^{4}-\frac{b}{(p-1)^{2}} \xi^{2}\right) s^{-2}\right)\right| \leq C s^{-3} \\
& \left|\left(h_{2},\left(W+\frac{p b \xi^{2}}{(p-1)^{2} s}\right) \eta_{0}\right)\right| \leq C s^{-3} \\
& \left|\left(h_{2}, M\left(\eta_{0}\right)\right)\right| \leq C s^{-3}
\end{aligned}
$$

because the only term in $s^{-2}$ coming from $M\left(\eta_{0}\right)$ is constant, i.e. is orthogonal to $h_{2}$. Now, we compute $\left(h_{2}, \xi^{2}\right)=\left\|h_{2}\right\|^{2}=8,\left(h_{2}, \partial^{2} \xi^{4}\right)=96$. Thus, all terms of order $s^{-2}$ will cancel if the following equation holds:

$$
c_{p}\left(\frac{96 p b^{2}}{2(p-1)^{4}}-\frac{8 b}{(p-1)^{2}}\right)=\frac{8 p b a}{(p-1)^{2}}
$$

Using our choice of $\mathrm{a}$, this is an equation for $\mathrm{b}$, whose solution is $b^{*}=\frac{(p-1)^{2}}{4 p}$. So, (105) holds.

The bounds $(106,107)$ are rather trivial. Since $\alpha$ is a smooth function of $\xi s^{-1 / 2}$, with bounded derivatives, we would get a bound with $s^{-3 / 2}$ in (106) from a Taylor expansion. 
But we have an extra $s^{-1}$ factor coming either from $\eta_{0}$ or from derivatives; (107) is proven by inspection.

Proof of Lemma 3. From (1.11), (2.5) together with (2.43), we get

$$
|N(\psi(\xi, s))| \leq C\left(|\psi(\xi, s)|^{\tilde{p}}+e^{-c s}\right) .
$$

where $\tilde{p}=\min (p, 2)>1$. Equations (21), (24), (25) and

$$
s^{-2+\delta}\left(1+|\xi|^{3}\right) \chi_{s} \leq C \tau^{-\frac{1}{2}+\delta}
$$

which holds for $\sigma \leq s, \tau \leq \sigma+\rho$, imply, for $\psi \in B_{0}$,

$$
\|\psi(\cdot, s)\|_{\infty} \leq C \tau^{-1 / 2+\delta}
$$

So, we have, using $K_{\tau-s}\left(\xi, \xi^{\prime}\right) \leq C e^{(\tau-s) \mathcal{L}}\left(\xi, \xi^{\prime}\right)$ (from $(41)$ and $\left.(84)\right)$ and $\mathcal{L} 1=1$ for the second term in (114),

$$
|\mathcal{N}(\psi)(\xi, \tau)| \leq C\left(\left(\tau^{-\frac{1}{2}+\delta}\right)^{\tilde{p}-1} \int_{\sigma}^{\tau} d s \int d \xi^{\prime} K_{\tau-s}\left(\xi, \xi^{\prime}\right)\left|\psi\left(\xi^{\prime}, s\right)\right|+(\tau-\sigma) e^{(\tau-\sigma)} e^{-c \tau}\right) .
$$

We want to show that

$$
\left|K_{\tau-s}\right| \psi||_{\tau} \leq C e^{(\tau-s)}|\psi|_{s}
$$

Write $|\psi|=\left|\psi \chi_{s}\right|+\left|\psi\left(1-\chi_{s}\right)\right|$ and estimate in (118) separately the large $\xi$ and the small $\xi$ parts of the norm $(21)$. We have, using $K_{\tau-s}\left(\xi, \xi^{\prime}\right) \leq C e^{(\tau-s) \mathcal{L}}\left(\xi, \xi^{\prime}\right)$ (coming from (84)), and (48),

$$
\left|K_{\tau-s}\right| \psi \chi_{s}|| \leq C e^{(\tau-s)} \tau^{-2+\delta}\left(1+|\xi|^{3}\right)|\psi|_{s}
$$

while, for $\chi_{\tau} K_{\tau-s}\left|\psi\left(1-\chi_{s}\right)\right|$, we can use a bound like (75). This proves (118) for $|\xi| \leq 2 K \tau^{1 / 2}$. For large $|\xi|$, we can use $(78,80)$ and the bound (116).

Now, for $\delta$ small and $\tau$ large (so that $C\left(\tau^{-\frac{1}{2}+\delta}\right)^{\tilde{p}-1} e^{\rho} \leq \tau^{-2 \delta}$ ), we get from $(117,118)$, and (23-25),

$$
\left\|\mathcal{S}(\psi)-\psi^{(0)}\right\|_{\rho}=\|\mathcal{N}(\psi)\|_{\rho} \leq \rho \tau^{-2 \delta}
$$

as required. The proof of (26) is similar.

To prove of $(28,29)$, write $N=\chi_{s} N+\left(1-\chi_{s}\right) N$, and use instead of (114)

$$
\left|\chi_{s} N(\psi(\xi, s))\right| \leq C\left(\chi_{s}|\psi(\xi, s)|^{2}+e^{-c s}\right) .
$$

because, for $|\xi|$ small, we can Taylor-expand $N$. By $(21,23-25)$, we have $|\chi \psi|^{2} \leq$ $C \tau^{-4+2 \delta}\left(1+|\xi|^{6}\right)$. Now, using $K_{\tau-s}\left(\xi, \xi^{\prime}\right) \leq C e^{(\tau-s) \mathcal{L}}\left(\xi, \xi^{\prime}\right)$, Lemmas 4 and $(2.7)$, we get $(28,29)$ for $\chi_{s} N$. For $\left(1-\chi_{s}\right) N$, we use the bound (114) on $N$ and $(39,51)$. The proof of $(30,31)$ follows immediately from (119) and the definition of the norm (where we can of course replace $\rho$ by $\tau-\sigma$ ). 


\section{The proof, $k>1$}

We need to study the equation $(2.2)$ with $F=0$ :

$$
\dot{\varphi}=L_{\tau}^{-2} \varphi^{\prime \prime}-\frac{1}{2 k} \xi \varphi^{\prime}-\frac{1}{p-1} \varphi+\varphi^{p}
$$

and with initial data as in (1.14):

$$
\varphi\left(\xi, \tau_{0}\right)=f_{b}^{*}(\xi)\left(1+\sum_{i=0}^{2 k-1} d_{i} \xi^{i}\left(p-1+b \xi^{2 k}\right)^{-1}\right)+g(\xi)
$$

We want to prove the

Theorem 4. There exist $\bar{\tau}<\infty$ and $\varepsilon>0$ such that for $\tau_{0}>\bar{\tau}$ and $g$ in $C^{0}(\mathbf{R})$ with $\|g\|_{\infty}<\varepsilon$ there are constants $d_{i} \in \mathbf{R}$ such that the equation (1) with the initial data (2) has a unique classical solution, which satisfies

$$
\left\|\varphi(\cdot, \tau)-f_{b^{*}}^{*}(\cdot)\right\|_{\infty} \rightarrow 0
$$

as $\tau \rightarrow \infty$, for some $b^{*}>0$, where $b^{*} \rightarrow b$ as $\varepsilon \rightarrow 0$ and $\tau_{0} \rightarrow \infty$.

We reduce the proof of the Theorem again to proving certain inductive properties of $\varphi$ as we increase $\tau$ in discrete units. First we introduce the deviation of $\varphi$ from $f_{b}$. It is convenient to write this in the form

$$
\varphi(\xi, \tau)=f_{b}^{*}(\xi)\left(1+e_{b}(\xi) \psi(\xi, \tau)\right)
$$

where we introduced

$$
e_{b}(\xi)=\frac{1}{p-1+b \xi^{2 k}}
$$

Then (1) is equivalent to

$$
\dot{\psi}=\mathcal{L}_{\tau} \psi+N(\psi)+D_{\tau}\left(\psi^{\prime}\right)+P_{\tau}(\psi)
$$

where

$$
\mathcal{L}_{\tau}=L_{\tau}^{-2} \partial_{\xi}^{2}-\frac{1}{2 k} \xi \partial_{\xi}+1,
$$

the nonlinear term is given by

$$
N(\psi)=\left(1+e_{b} \psi\right)^{p}-1-p e_{b} \psi
$$

and

$$
\begin{aligned}
D_{\tau}\left(\psi^{\prime}\right) & =-\frac{4 p k b}{p-1} L_{\tau}^{-2} e_{b} \xi^{2 k-1} \psi^{\prime} \\
P_{\tau}(\psi) & =L_{\tau}^{-2} \xi^{2 k-2} e_{b}(\xi)\left(\alpha_{1}+\alpha_{2} \xi^{2 k} e_{b}+\left(\alpha_{3}+\alpha_{4} \xi^{2 k} e_{b}\right) \psi\right) .
\end{aligned}
$$


where $\alpha_{i}=\alpha_{i}(k, p, b)$ are constants. Note that, as opposed to (3.41), there is no potential in (7). This will greatly simplify the analysis, see Lemma 1 below. Due to the factor $L_{\tau}^{-2}$, the $D_{\tau}, P_{\tau}$ terms will be small, like the "irrelevant" $F$ in Section 3 .

Let

$$
M=\frac{2 k p}{p-1}
$$

and consider $\psi(\sigma)$ of the form

$$
\psi(\sigma)=\sum_{m=0}^{[M]} \psi_{m}(\sigma) h_{m}(\cdot, \sigma)+\psi^{\perp}(\sigma) \equiv \psi^{<}+\psi^{\perp}
$$

where [.] denotes the integer part and $\psi^{\perp}$ is $C^{0}$ in $\xi$, is bounded by

$$
\left|\psi^{\perp}(\sigma)\right|_{\sigma} \equiv \sup _{\xi}\left(L_{\sigma}^{-M}+|\xi|^{M}\right)^{-1}\left|\psi^{\perp}(\xi, \sigma)\right| \leq \varepsilon(\sigma)
$$

and is orthogonal in $L^{2}\left(\mathbf{R}, d \mu_{\sigma}\right)$ to $h_{m}(\sigma)$ with $m \leq[M]$, where the scalar product is defined by (2.32). Also assume

$$
\left|\psi_{m}(\sigma)\right| \leq \begin{cases}\epsilon(\sigma) & m \neq 2 k \\ \epsilon(\sigma)^{3 / 2} & m=2 k\end{cases}
$$

and take

$$
\varepsilon(\sigma)=L_{\sigma}^{-\delta}
$$

for $\delta>0$. The reason for choosing $M$ larger than $2 k$ is that the integration by parts works only for such large $M$, see Lemma 1 below. However, to control the nonlinear term (8), we cannot take $M$ too large. We will need $N(\psi)$ to be bounded by $|\xi|^{M}$. But, with the choice $(11),\left(e_{b} \psi\right)^{p} \leq C|\xi|^{(M-2 k) p}=C|\xi|^{M}$. We have then the

Proposition. Given $\rho>0$, there exist $\delta>0$ and $\bar{\tau}<\infty$ such that if $\sigma>\bar{\tau}$ and $\psi(\sigma)$ satisfies (12)-(14) then the equation (6) has a unique classical solution, for $\tau \in[\sigma, \sigma+\rho]$ which can be expressed in the form (12) with

$$
\begin{gathered}
\left|\psi_{m}(\tau)-e^{(\tau-\sigma)\left(1-\frac{m}{2 k}\right)} \psi_{m}(\sigma)\right| \leq(\tau-\sigma) C(\rho) \varepsilon(\sigma)^{2} \\
\left|\psi^{\perp}(\tau)\right|_{\tau} \leq C e^{-\frac{1}{p-1}(\tau-\sigma)} \varepsilon(\sigma) .
\end{gathered}
$$

Here and below, $C(\rho) \leq C e^{c \rho}$. We will now prove the Theorem, given the Proposition.

Proof of Theorem 4. Let $\varphi\left(\xi, \tau_{0}\right)$ be given by (2). We put (see (4))

$$
\psi\left(\xi, \tau_{0}\right)=\sum_{i=0}^{2 k-1} d_{i} \xi^{i}+\left(p-1+b \xi^{2 k}\right)^{\frac{p}{p-1}} g(\xi)=\sum_{m=0}^{[M]} \psi_{m}\left(\tau_{0}\right) h_{m}\left(\xi, \tau_{0}\right)+\psi^{\perp}\left(\xi, \tau_{0}\right)
$$

and get from (2.30)-(2.32),

$$
\left|\psi_{m}\left(\tau_{0}\right)-d_{m}\right| \leq C\left(L_{\tau_{0}}^{-1}+L_{\tau_{0}}^{m} \varepsilon\right) \quad m<2 k
$$




$$
\left|\psi_{m}\right| \leq C L_{\tau_{0}}^{m} \varepsilon \quad m \geq 2 k
$$

and, since

$$
\psi^{\perp}=\gamma-\sum_{m=0}^{[M]} h_{m}\left(\gamma, h_{m}\right)_{\tau_{0}}\left(h_{m}, h_{m}\right)_{\tau_{0}}^{-1}
$$

with $\gamma=\left(p-1+b \xi^{2 k}\right)^{\frac{p}{p-1}} g$, we find

$$
\begin{aligned}
\left|\psi^{\perp}\left(\xi, \tau_{0}\right)\right| & \leq C \varepsilon\left[1+|\xi|^{M}+\sum_{m=0}^{[M]}\left(L_{\tau_{0}}^{-m}+|\xi|^{m}\right) L_{\tau_{0}}^{m}\right] \\
& \leq C \varepsilon L_{\tau_{0}}^{M}\left(L_{\tau_{0}}^{-M}+|\xi|^{M}\right)
\end{aligned}
$$

where we use, see (2.30),

$$
\left|h_{m}(\xi, \tau)\right| \leq C\left(L_{\tau}^{-m}+|\xi|^{m}\right) .
$$

Therefore, given $\tau_{0}$, we may, by taking $\varepsilon$ small, satisfy (12)-(14) for $\sigma=\tau_{0}$. Moreover, as in Section 3, instead of varying $d_{m}$ in (1.14), we can vary $\psi_{m}\left(\tau_{0}\right), m<2 k$, in (17), in the interval defined by (14) with $\sigma=\tau_{0}$. Put now $\tau_{n}=\tau_{0}+n \rho$, and write

$$
\varphi\left(\xi, \tau_{n}\right)=f_{b_{n}}(\xi)\left(1+e_{b_{n}}(\xi) \psi\left(\xi, \tau_{n}\right)\right)
$$

with $\psi$ satisfying (12)-(14) with $\sigma=\tau_{n}$. By the Proposition,

$$
\varphi\left(\xi, \tau_{n+1}\right)=f_{b_{n}}(\xi)\left(1+e_{b_{n}}(\xi) \tilde{\psi}\left(\xi, \tau_{n+1}\right)\right)
$$

with $\tilde{\psi}$ satisfying

$$
\left|\tilde{\psi}_{m}-e^{\rho(1-m / 2 k)} \psi_{m}\right| \leq C(\rho) \rho \varepsilon\left(\tau_{n}\right)^{2}
$$

and

$$
\left|\tilde{\psi}^{\perp}\right|_{\tau_{n+1}} \leq C e^{-\frac{\rho}{p-1}} \varepsilon\left(\tau_{n}\right) .
$$

Put now

$$
b_{n+1}=b_{n}-\tilde{\psi}_{2 k}
$$

whereby, for $\delta$ small enough in the definition of $\epsilon(\sigma)$,

$$
\varphi\left(\xi, \tau_{n+1}\right)=f_{b_{n+1}}(\xi)\left(1+e_{b_{n+1}}(\xi) \bar{\psi}(\xi, \tau)\right)
$$

and

$$
\begin{aligned}
& \left|\bar{\psi}_{m}-e^{\rho(1-m / 2 k)} \psi_{m}\right| \leq C(\rho) \rho \varepsilon\left(\tau_{n}\right)^{2} \quad m<2 k \\
& \left|\bar{\psi}_{2 k}\right| \leq C \rho \varepsilon\left(\tau_{n}\right)^{2}<\varepsilon\left(\tau_{n+1}\right)^{\frac{3}{2}} \\
& \left|\bar{\psi}_{m}\right| \leq \varepsilon\left(\tau_{n+1}\right) \quad m>2 k \\
& \left|\bar{\psi}^{\perp}\right|_{\tau_{n+1}} \leq \varepsilon\left(\tau_{n+1}\right) .
\end{aligned}
$$

We use $C(\rho) \varepsilon\left(\tau_{n}\right)^{2} \leq \varepsilon\left(\tau_{n+1}\right)$ and $C(\rho) \varepsilon\left(\tau_{n}\right)^{\frac{3}{2}} \leq \varepsilon\left(\tau_{n+1}\right)$. Moreover, by the same topological argument as in Section 3, we now establish the existence of $\psi_{m}\left(\tau_{0}\right)$ for $m<2 k$, such that $\psi$ in (23) satisfies (12)-(14) for all $n$. The contrary assumption would now 
allow us to construct a homotopy between the identity and constant maps respectively from $S^{2 k} \rightarrow S^{2 k}$. From $(26,24,14)$ we then deduce that

$$
b_{n} \underset{n \rightarrow \infty}{\longrightarrow} b^{*}
$$

and $\left|b^{*}-b_{0}\right| \leq C \varepsilon\left(\tau_{0}\right)^{\frac{3}{2}}$, with $b_{0}=b$, implies that $b^{*} \rightarrow b$ as $\varepsilon \rightarrow 0$ and $\tau_{0} \rightarrow \infty$ (see (18, $19,21))$.

We now turn to the proof of the Proposition. We consider the following integral equation related to our PDE (6):

$$
\psi(\tau)=K_{\tau \sigma} \psi(\sigma)+\int_{\sigma}^{\tau} d s K_{\tau s}\left(N(\psi(s))+P_{s}(\psi(s))\right)+\mathcal{D}(\psi, \tau)
$$

where

$$
\mathcal{D}(\psi, \tau)=4 p k b(p-1)^{-1} \int_{\sigma}^{\tau} d s L_{s}^{-2} \int d \xi^{\prime} \partial_{\xi^{\prime}}\left(K_{\tau s}\left(\xi, \xi^{\prime}\right) e_{b}\left(\xi^{\prime}\right) \xi^{\prime 2 k-1}\right) \psi\left(\xi^{\prime}, s\right)
$$

A classical solution of (6) satisfies (32). Note that $\mathcal{D}(\psi, \tau)$ is obtained by integration by parts from the term that naturally follows from the integration of (6). This form is more convenient for us since we want to work with $C^{0}$ data. We show that (32) has a unique solution in a suitable space and that this solution is the classical solution of (6).

Let us introduce the following norms in $C^{0}(\mathbf{R})$. We write $\psi \in C^{0}(\mathbf{R})$ as in (12)

$$
\psi(\xi)=\sum_{m=0}^{[M]} \psi_{m} h_{m}(\xi, \sigma)+\psi^{\perp}(\xi)
$$

and let

$$
\|\psi\|_{\sigma}=\sum_{m=0}^{[M]}\left|\psi_{m}\right|+\left|\psi^{\perp}\right|_{\sigma} .
$$

with $\left|\psi^{\perp}\right|_{\sigma}$ defined in (13). It is straightforward to check that

$$
C_{1}(\sigma)\|\psi\| \leq\|\psi\|_{\sigma} \leq C_{2}(\sigma)\|\psi\|
$$

with $C_{i}>0$ and

$$
\|\psi\|=\sup _{\xi}\left(1+|\xi|^{M}\right)^{-1}|\psi(\xi)|,
$$

so $C^{0}(\mathbf{R})$ is complete in the norm $\|\cdot\|_{\sigma}$.

Write (32) as

$$
\psi(\tau)=\psi^{0}(\tau)+\mathcal{N}(\psi, \tau)+\mathcal{P}(\psi, \tau)+\mathcal{D}(\psi, \tau)
$$

with (using (2.33)),

$$
\psi^{0}(\tau)=K_{\tau \sigma} \psi(\sigma)=\sum_{m=0}^{[M]} e^{(\tau-\sigma)\left(1-\frac{m}{2 k}\right)} \psi_{m} h_{m}(\xi, \tau)+K_{\tau \sigma} \psi^{\perp} .
$$


Let us first bound $\psi^{0}$ :

Lemma 1. We have

$$
\left\|K_{\tau \sigma} \psi^{\perp}\right\|_{\tau} \leq C e^{-\frac{1}{p-1}(\tau-\sigma)}\left\|\psi^{\perp}\right\|_{\sigma} .
$$

Proof We use the conjugation (2.24): let

$$
\theta(\xi)=\psi^{\perp}\left(L_{\sigma}^{-1} \xi\right), \tilde{\theta}(\xi)=\left(K_{\tau \sigma} \psi^{\perp}\right)\left(L_{\tau}^{-1} \xi\right)
$$

whence

$$
\tilde{\theta}=e^{(\tau-\sigma) \mathcal{L}} \theta
$$

and (see $(35,13))$,

$$
|\theta(\xi)| \leq L_{\sigma}^{-M}\left(1+|\xi|^{M}\right)\left\|\psi^{\perp}\right\|_{\sigma}
$$

with

$$
\left(\theta, h_{m}\right)=0 \quad m \leq[M],
$$

where the scalar product now is given by (2.10). Proceeding as in the derivation of (3.67), we get, for $\tau-\sigma \geq 1$,

$$
\begin{aligned}
\left|\left(e^{(\tau-\sigma) \mathcal{L}} \theta\right)(\xi)\right| & =\left|\int \partial_{\xi^{\prime}}^{[M]+1} N\left(\xi, \xi^{\prime}\right) f^{(-[M]-1)}\left(\xi^{\prime}\right) d \xi^{\prime}\right| \\
& \leq C e^{(\tau-\sigma)} e^{-([M]+1) \frac{\tau-\sigma}{2}} L_{\sigma}^{-M}\left(1+|\xi|^{M}\right)\left\|\psi^{\perp}\right\|_{\sigma}
\end{aligned}
$$

where $N\left(\xi, \xi^{\prime}\right)$ is defined in (3.63), $f(\xi)=e^{-\xi^{2} / 4} \theta(\xi)$ and we used the analogue of Lemma 3.6, i.e.

$$
\left|f^{(-m)}(\xi)\right| \leq C e^{-m \frac{\tau-\sigma}{2}} L_{\sigma}^{-M}(1+|\xi|)^{M-m}\left\|\psi^{\perp}\right\|_{\sigma}
$$

for $0 \leq m \leq[M]+1$, and Lemma 3.4. Since by (11) and (2.4),

$$
e^{-\frac{M}{2}(\tau-\sigma)} L_{\sigma}^{-M}=e^{-\frac{p}{p-1}(\tau-\sigma)} L_{\tau}^{-M}
$$

we get

$$
\left|K_{\tau \sigma} \psi^{\perp}(\xi)\right| \leq C e^{-\frac{(\tau-\sigma)}{p-1}}\left(L_{\tau}^{-M}+|\xi|^{M}\right)\left\|\psi^{\perp}\right\|_{\sigma} .
$$

For $\tau-\sigma<1$ we need not integrate by parts and the bound follows using (3.48).

In particular, from Lemma 1 and $(39,13,14)$ we deduce

$$
\left\|\psi^{0}\right\|_{\tau} \leq C(\rho) \varepsilon(\sigma)
$$

To solve (32), consider the ball

$$
\mathcal{B}=\left\{\psi \in C^{0}(\mathbf{R} \times[\sigma, \sigma+\rho]) \mid \sup _{\tau \in[\sigma, \sigma+\rho]}\left\|\psi-\psi^{0}\right\|_{\tau} \leq \epsilon(\sigma)^{\frac{1+\tilde{p}}{2}}\right\}
$$

where $\tilde{p}=\min (p, 2)$. Note that, for $\psi \in \mathcal{B}$, we have, for $s \in[\sigma, \sigma+\rho]$

$$
\begin{aligned}
& \left|\psi_{m}(s)\right| \leq C(\rho) \epsilon(\sigma) \\
& \left|\psi^{\perp}(s)\right| \leq C(\rho) \epsilon(\sigma)\left(L_{s}^{-M}+|\xi|^{M}\right)
\end{aligned}
$$


We have then

Lemma 2. Equation (32) has a unique solution $\psi \in \mathcal{B}$. The properties (15) and (16) hold for $\psi$.

Proof of Lemma 2. We shall use the contraction mapping principle. Let us first bound the nonlinear term

$$
\begin{aligned}
\mathcal{N}(\xi, \tau) & =\int_{\sigma}^{\tau} d s \int d \xi^{\prime} K_{\tau s}\left(\xi, \xi^{\prime}\right) N\left(\psi(s), \xi^{\prime}\right) \\
& =\sum_{m=0}^{[M]} \mathcal{N}_{m}(\tau) h_{m}(\xi, \tau)+\mathcal{N}^{\perp}(\xi, \tau)
\end{aligned}
$$

Here (use (2.33))

$$
\mathcal{N}_{m}(\tau)=\int_{\sigma}^{\tau} d s e^{(\tau-s)\left(1-\frac{m}{2 k}\right)} \frac{\left(h_{m}, N\right)_{s}}{\left(h_{m}, h_{m}\right)_{s}} .
$$

and

$$
\mathcal{N}^{\perp}(\cdot, \tau)=\int_{\sigma}^{\tau} d s K_{\tau s} N^{\perp}(\psi(s))
$$

Consider first $\mathcal{N}_{m}(\tau)$. Let $\chi(\xi)=\chi(|\xi| \leq 1)$ and $\chi^{c}=1-\chi$ and insert

$$
N=\chi N+\chi^{c} N
$$

in (48). From (8) and the bounds (46) on $\psi$, we have

$$
\left|\chi^{c} N\right| \leq \chi^{c}\left(C(\rho) \epsilon(\sigma) e_{b}|\xi|^{M}\right)^{p} \leq(C(\rho) \epsilon(\sigma))^{p}|\xi|^{M}
$$

(use (5) and recall that $M=2 k p(p-1)^{-1}$ ) and therefore from (2.32)

$$
\left|\frac{\left(h_{m}, \chi^{c} N\right)_{s}}{\left(h_{m}, h_{m}\right)_{s}}\right| \leq e^{-L_{\tau}^{2} / c} \leq \epsilon(\sigma)^{2} .
$$

As for $\chi N$, Taylor expanding, we obtain

$$
\chi N=\sum_{j=2}^{K} c_{j} \chi\left(e_{b} \psi\right)^{j}+R_{K}
$$

with the bound for the remainder

$$
\left|R_{K}\right| \leq C \chi\left|e_{b} \psi\right|^{K+1} \leq(C(\rho) \epsilon(\sigma))^{K+1} .
$$

Thus,

$$
\left|\frac{\left(h_{m}, R_{K}\right)_{s}}{\left(h_{m}, h_{m}\right)_{s}}\right| \leq L_{s}^{m}(C(\rho) \epsilon(\sigma))^{K+1} \leq \epsilon(\sigma)^{2}
$$

provided we take $K>K(\delta)$. 
For the sum in (52) insert the decomposition (12):

$$
\chi\left(e_{b} \psi\right)^{j}=\chi\left(e_{b} \psi^{<}\right)^{j}+S_{j}
$$

and, by the bound (46) for $\psi^{\perp}$, we get, for $S=\sum_{j=2}^{K} c_{j} S_{j}$

$$
|S| \leq C(\rho) \epsilon(\sigma)^{2} \chi(\xi)\left(L_{\tau}^{-M}+|\xi|^{M}\right) .
$$

Thus

$$
\left|\frac{\left(h_{m}, S\right)_{s}}{\left(h_{m}, h_{m}\right)_{s}}\right| \leq C(\rho) L_{s}^{m-M} \epsilon(\sigma)^{2} \leq C(\rho) \epsilon(\sigma)^{2} .
$$

For the first term in (55), we insert

$$
e_{b}=(p-1)^{-1}\left(\sum_{l=0}^{L}\left(-\frac{b \xi^{2 k}}{p-1}\right)^{l}+\left(-\frac{b \xi^{2 k}}{p-1}\right)^{L+1} e_{b}(\xi)\right)
$$

and the expansion (12) for $\psi^{<}$, to get

$$
A \equiv \sum_{j=2}^{K} c_{j} \chi\left(e_{b} \psi^{<}\right)^{j}=\sum_{\mathbf{n}, p} c_{\mathbf{n}, p} \chi \xi^{p} \prod_{i=1}^{[M]} \psi_{i}^{n_{i}} h_{i}^{n_{i}}+\epsilon(\sigma)^{2} \xi^{2 k(L+1)} \chi Q \equiv A_{1}+A_{2}
$$

where $\mathbf{n}=\left(n_{1}, \ldots, n_{[M]}\right), \sum n_{i} \geq 2$ and $\chi Q$ is bounded.

For $A_{1}$, put $\chi=1-\chi^{c}$, estimate the $\chi^{c}$-term by (51) and use

$$
\left|\frac{\left(h_{m}, \xi^{p} \prod h_{i}^{n_{i}}\right)_{s}}{\left(h_{m}, h_{m}\right)_{s}}\right|= \begin{cases}0 & p+\sum n_{i}<m \\ \leq C L_{s}^{m-p-\sum n_{i}} & p+\sum n_{i} \geq m\end{cases}
$$

and (46), to get

$$
\left|\frac{\left(h_{m}, A_{1}\right)_{s}}{\left(h_{m}, h_{m}\right)_{s}}\right| \leq C(\rho) \epsilon(\sigma)^{2} .
$$

For $A_{2}$, take $2 k(L+1) \geq M$ to obtain (61) again, for $A_{2}$. Thus, inserting $(51,54,57$, 61) into (48),

$$
\left|\mathcal{N}_{m}(\tau)\right| \leq(\tau-\sigma) C(\rho) \epsilon(\sigma)^{2}
$$

for $\tau \in[\sigma, \sigma+\rho]$.

For $\mathcal{N}^{\perp}$ in $(47,49)$, we proceed in a similar fashion; (50) yields

$$
\left|\left(\chi^{c} N\right)^{\perp}\right| \leq(C(\rho) \epsilon(\sigma))^{p}\left(L_{\tau}^{-M}+|\xi|^{M}\right)
$$

using $X^{\perp}=X-\sum_{0}^{[M]} h_{m}\left(h_{m}, h_{m}\right)_{s}^{-1}\left(h_{m}, X\right)_{s},(3.74)$, and the first inequality in (51).

For $(\chi N)^{\perp}$, we write as in $(52,55,59)$,

$$
\chi N=A+S+R_{K} .
$$

$S$ has the bound (56), and, from (53), we get, for $K$ large,

$$
\left|R_{K}\right| \leq \epsilon(\sigma)^{2} L_{\sigma}^{-M}
$$


As for $A$, by (59), $A_{2}$ satisfies a bound like (56) and for $A_{1}$ we divide the sum into $p+\sum n_{i}>[M]$ and $\leq[M]$. For the first sum,

$$
\chi\left|\xi^{p} \prod_{i=1}^{[M]} h_{i}^{n_{i}}\right| \leq C\left(L_{s}^{-M}+|\xi|^{M}\right)
$$

since $h_{i}$ is bounded by (22), and, for the second sum, replace $\chi=1-\chi^{c}$ by $-\chi^{c}$ since 1 will not contribute to $A^{\perp}\left(\left(\xi^{p} \prod_{i=1}^{[M]} h_{i}^{n_{i}}\right)^{\perp}=0\right.$ for these terms $)$. Since $|\xi| \geq 1$, in this case

$$
\chi^{c}\left|\xi^{p} \prod_{i=1}^{[M]} h_{i}^{n_{i}}\right| \leq C|\xi|^{M}
$$

and altogether

$$
\left|A^{\perp}\right| \leq C(\rho) \epsilon(\sigma)^{2}\left(L_{s}^{-M}+|\xi|^{M}\right)
$$

(we used again the fact that this bound for $A$ implies it for $A^{\perp}$ ). By $(63),(65),(68)$,

$$
\left|N^{\perp}\right| \leq C(\rho)\left(\epsilon(\sigma)^{2}+\epsilon(\sigma)^{p}\right)\left(L_{s}^{-M}+|\xi|^{M}\right)
$$

and therefore, using $(41,42)$ and Lemma 3.4,

$$
\left|\mathcal{N}^{\perp}\right| \leq C(\rho)(\tau-\sigma)\left(\epsilon(\sigma)^{2}+\epsilon(\sigma)^{p}\right)\left(L_{\sigma}^{-M}+|\xi|^{M}\right) .
$$

We may bound $C(\rho)(\tau-\sigma)\left(\epsilon(\sigma)^{2}+\epsilon(\sigma)^{p}\right)$ by $\epsilon(\sigma)^{\frac{\tilde{p}+1}{2}}$.

Next, we consider $\mathcal{P}$ in (38). From (10) we have

$$
\left|P_{\tau} \chi^{c}\right| \leq C L_{\tau}^{-2}|\xi|^{M-2}
$$

which fits to our bounds (see (63)). The $|\xi|<1$ analysis proceeds as above.

Finally, for the $\mathcal{D}$ term in (33), the terms where $\partial_{\xi^{\prime}}$ does not act on $K$ are estimated as above and we write the remaining term as $4 p k b(p-1)^{-1} F$ with

$$
F(\xi)=\int_{\sigma}^{\tau} d s L_{s}^{-2} \int d \xi^{\prime} e^{\frac{(\tau-s)}{2 k}} \partial_{\xi} K_{\tau s}\left(\xi, \xi^{\prime}\right) \rho\left(\xi^{\prime}, s\right)=\sum_{m=0}^{[M]} F_{m} h_{m}+F^{\perp}
$$

where $\rho\left(\xi^{\prime}, s\right)=\left(\xi^{\prime}\right)^{2 k-1} e_{b}\left(\xi^{\prime}\right) \psi\left(\xi^{\prime}, s\right)$, and where we used $(2.27,2.28)$ to change $\partial_{\xi^{\prime}}$ into $\partial_{\xi}$. We write again

$$
\rho=\sum_{m=0}^{[M]} \rho_{m} h_{m}+\rho^{\perp}
$$

and we have from (46),

$$
\|\rho(s)\|_{s} \leq C(\rho) \epsilon(\sigma) .
$$

Since $\partial_{\xi} h_{m}=m h_{m-1}$ and the adjoint of $\partial_{\xi}$ in $(\cdot, \cdot)_{\tau}$ is $-\partial_{\xi}+\frac{L_{\tau}}{2} \xi$, we have

$\left|F_{m}(\tau)\right| \leq(m+1) \int_{\sigma}^{\tau} d s L_{s}^{-2} e^{\left(1-\frac{m}{2 k}\right)(\tau-s)}\left|\rho_{m+1}(s)\right|+(\tau-\sigma) C(\rho) L_{\sigma}^{-1}\left(h_{m}, \xi K_{\tau s} \rho^{\perp}\right)_{\tau}\left(h_{m}, h_{m}\right)_{\tau}^{-1}$. 
Therefore

$$
\left|F_{m}(\tau)\right| \leq(\tau-\sigma) C(\rho) L_{\sigma}^{-1} \epsilon(\sigma) \leq(\tau-\sigma) \epsilon(\sigma)^{2} .
$$

As for $F^{\perp}$, we have

$$
F^{\perp}=\int_{\sigma}^{\tau} d s L_{s}^{-2} e^{\frac{(\tau-s)}{2 k}} \partial K_{\tau s} \rho(s)^{\perp}
$$

and we need the bound for $\partial K$, derived from $(2.27,2.28)$ :

$$
\left|\partial_{\xi} K_{\tau s}\left(\xi, \xi^{\prime}\right)\right| \leq C(\rho) L \delta_{\frac{1}{2} L^{2}}\left(e^{\frac{s-\tau}{2 k}} \xi-\xi^{\prime}\right) \leq \frac{C(\rho) L_{\tau}}{\sqrt{\tau-s}} \delta_{\frac{1}{2} L^{2}}\left(e^{\frac{s-\tau}{2 k}} \xi-\xi^{\prime}\right)
$$

(where, recall, $L^{2}=L_{s}^{2}\left(1-e^{s-\tau}\right)^{-1}$ ). This implies

$$
\left|F^{\perp}\right|_{\tau} \leq C(\rho) L_{\sigma}^{-1} \epsilon(\sigma) \leq \epsilon(\sigma)^{2}
$$

The bounds $(62,70),(76,79)$, and similar bounds for the other terms in $\mathcal{P}$, $\mathcal{D}$, imply that the RHS of (32) maps $\mathcal{B}$ into itself. That this map also contracts in this ball is showed in a similar fashion. Finally, (15) follows from $(32,39,62,76)$ and (16) follows from $(32,40,70,79)$, using $p>1$ in $(70)$ to bound its RHS by $e^{-\frac{\rho}{p-1}} \epsilon(\sigma)\left(L_{\tau}^{-N}+|\xi|^{N}\right)$.

To conclude the proof of the Proposition, we need to show that the $\psi$ as constructed above actually is $C^{2}$ in $\xi$ and $C^{1}$ in $\tau$ on $(\sigma, \sigma+\rho]$.

For smoothness, the idea is to improve the smoothness of $\psi$ iteratively using (32) and the regularity properties of the kernel $K$. This is completely straightforward, but we will sketch the argument here for completeness. Let us consider the least regular term in (32) i.e. the $F$ term in (72). The bound (78) may be improved to

$$
\left|\partial_{\xi} K_{\tau s}\left(\xi_{1}, \xi^{\prime}\right)-\partial_{\xi} K_{\tau s}\left(\xi_{2}, \xi^{\prime}\right)\right| \leq \frac{C(\rho) L_{\tau}^{2-\eta}\left|\xi_{1}-\xi_{2}\right|^{1-\eta}}{(\tau-s)^{1-\frac{\eta}{2}}}\left(\delta_{\frac{1}{2} L^{2}}\left(e^{\frac{s-\tau}{2 k}} \xi_{1}-\xi^{\prime}\right)+\delta_{\frac{1}{2} L^{2}}\left(e^{\frac{s-\tau}{2 k}} \xi_{2}-\xi^{\prime}\right)\right)
$$

for $\eta>0$. This implies that $F$ is $C^{1-\eta}$ (Hölder continuous with exponent $1-\eta$ ). The other terms of $\mathcal{D}, \mathcal{N}$, and $\mathcal{P}$ are analyzed similarily to be $C^{2-\eta}$. Thus our solution $\psi \in C^{1-\eta}$, and, by considering the solution $\psi^{0}$ of the linear equation, we see that its $1-\eta$ Hölder norm is bounded by $\mathcal{O}\left((s-\sigma)^{-\frac{1-\eta}{2}}\right)$ for $s-\sigma$ small. With this knowledge we next prove that $F \in C^{2-\eta}$. Indeed,

$$
F(\xi)=\int_{\sigma}^{\tau} d s L_{s}^{-2} \int d \xi^{\prime} H\left(\xi, \xi^{\prime}\right)
$$

where, putting the derivative back on $\xi^{\prime}$, and using $\int d \xi^{\prime} \partial_{\xi^{\prime}} K_{\tau s}\left(\xi, \xi^{\prime}\right)=0$, we have

$$
H\left(\xi, \xi^{\prime}\right)=\partial_{\xi^{\prime}} K_{\tau s}\left(\xi, \xi^{\prime}\right)\left(\rho\left(\xi^{\prime}, s\right)-\rho\left(e^{\frac{\tau-s}{2 k}} \xi, s\right)\right)
$$

and we may now use the Hölder property of $\rho$ (for each power of $\left|\xi^{\prime}-e^{\frac{\tau-s}{2 k}} \xi\right|$, one gains a power of $|\tau-s|^{1 / 2}$ ) to bound

$$
\int d \xi^{\prime}\left|\partial_{\xi} H\left(\xi, \xi^{\prime}\right)\right| \leq C(\rho)(\tau-s)^{-\frac{1+\eta}{2}}(s-\sigma)^{-\frac{1-\eta}{2}}
$$


where the $(s-\sigma)^{-\frac{1-\eta}{2}}$ comes from the Hölder estimate on $\psi$. Now, (83) is integrable in $s$, for $\eta$ small. Thus $\psi$ is $C^{1}$. This allows us to integrate by parts and to write (33) as :

$$
\mathcal{D}(\psi, \tau)=-4 p k b(p-1)^{-1} \int_{\sigma}^{\tau} d s L_{s}^{-2} \int d \xi^{\prime} K_{\tau s}\left(\xi, \xi^{\prime}\right) e_{b}\left(\xi^{\prime}\right) \xi^{\prime 2 k-1} \partial_{\xi^{\prime}} \psi\left(\xi^{\prime}, s\right)
$$

and by the above argument to prove that $\mathcal{D}$ and hence $\psi$ is $C^{2-\eta}$.

Finally, we write (38), reasoning as in (82),

$$
\partial \psi(\xi, \tau)=\partial \psi^{0}(\xi, \tau)+\int d s \int d \xi^{\prime} \partial_{\xi} K_{\tau s}\left(\xi, \xi^{\prime}\right)\left(\Psi\left(\xi^{\prime}\right)-\Psi(\xi)\right)
$$

with $\Psi \in C^{1-\eta}$ and apply the argument above to conclude that $\psi$ is $C^{2}$. The derivative with respect to $\tau$ is similar. This concludes the proof of the Proposition.

\section{References}

[1] Barenblatt, G.I., Similarity, Self-Similarity and Intermediate Asymptotics, Consultants Bureau, New York, 1979.

[2] Bressan, A., Stable blow-up patterns, J. Diff. Eq, 98, 57-75 (1992).

[3] Bricmont, J., Kupiainen, A., Lin, G., Renormalization group and asymptotics of solutions of nonlinear parabolic equations, Comm.Pure.Appl.Math., to appear.

[4] Filippas, S., Kohn, R.V., Refined asymptotics for the blow-up of $u_{t}-\Delta u=u^{p}$, Comm. Pure Appl. Math, 45, 821-869 (1992).

[5] Filippas, S., Liu, W., On the blow-up of multidimensional semilinear heat equations, Ann. Inst. H. Poincaré, to appear.

[6] Glimm, J., Jaffe, A., Quantum Physics, Springer, New York, 1981.

[7] Goldenfeld, N., Martin, O., Oono, Y., Liu, F., Anomalous dimensions and the renormalization group in a nonlinear diffusion process, Phys. Rev. Lett. 64, 13611364 (1990).

[8] Goldenfeld, N., Martin, O., Oono, Y., Asymptotics of partial differential equations and the renormalization group, to appear in the Proc. of the NATO ARW on Asymptotics beyond all orders, edited by S. Tanveer (Plenum Press).

[9] Herrero, M.A., Velazquez, J.J.L., Some results on blow-up for semilinear parabolic problems. IMA preprint 1000.

[10] Herrero, M.A., Velazquez, J.J.L., Blow-up behaviour of one-dimensional semilinear parabolic equations, Ann. Inst. H. Poincaré, 10, 131-189 (1993).

[11] Herrero, M.A., Velazquez, J.J.L., Flat blow-up in one-dimensional semilinear heat equations, Differential and Integral Equations, 5, 973-997 (1992). 
[12] Herrero, M.A., Velazquez, J.J.L., Blow-up profiles in one-dimensional, semilinear parabolic problems, Comm. in P.D.E., 17, 205-219 (1992) .

[13] Levine, H.A., The role of critical exponents in blow-up theorems, SIAM Review, 32, 262-288 (1990).

[14] Simon, B., Functional Integration and Quantum Physics, Academic Press, New York, 1979.

[15] Velazquez, J.L.L., Higher dimensional blow-up for semilinear parabolic equations, IMA preprint 968. 